\title{
Notes on generalized global symmetries in QFT
}

\author{
Eric Sharpe \\ Department of Physics MC 0435 \\ 850 West Campus Drive \\ Virginia Tech \\ Blacksburg, VA 24061 \\ ersharpe@vt.edu
}

\begin{abstract}
It was recently argued that quantum field theories possess one-form and higher-form symmetries, labelled 'generalized global symmetries.' In this paper, we describe how those higher-form symmetries can be understood mathematically as special cases of more general 2 -groups and higher groups, and discuss examples of quantum field theories admitting actions of more general higher groups than merely one-form and higher-form symmetries. We discuss analogues of topological defects for some of these higher symmetry groups, relating some of them to ordinary topological defects. We also discuss topological defects in cases in which the moduli 'space' (technically, a stack) admits an action of a higher symmetry group. Finally, we outline a proposal for how certain anomalies might potentially be understood as describing a transmutation of an ordinary group symmetry of the classical theory into a 2 -group or higher group symmetry of the quantum theory, which we link to WZW models and bosonization.
\end{abstract}

August 2015 


\section{Contents}

1 Introduction

2 Overview of 2-groups and higher groups 5

3 Examples of higher group symmetries in QFT 10

3.1 Review of $q$-form symmetries in gauge theories . . . . . . . . . . . . 10

3.2 Gauge theories with subgroup-invariant massless matter . . . . . . . . . 12

3.3 Boundary structures in Dijkgraaf-Witten theory . . . . . . . . . . 16

3.4 WZW models . . . . . . . . . . . . . . . . . . . . . . 19

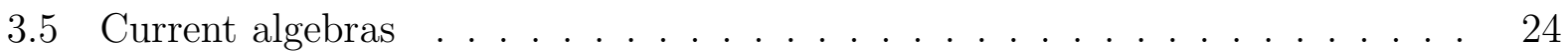

3.6 Other Kac-Moody actions . . . . . . . . . . . . . . . 25

4 Cosmological defects and generalized moduli spaces 25

4.1 Generalities on defects and ordinary moduli spaces . . . . . . . . . 25

4.2 Generalized moduli spaces . . . . . . . . . . . . . . . . 30

4.3 Analogues of Goldstone's theorem and generalized moduli spaces . . . . . . . 32

5 Anomalies as transmutation $\quad 34$

5.1 Two-dimensional cases ............................. 34

5.2 Conjectures on elliptic genera . . . . . . . . . . . . . . 36

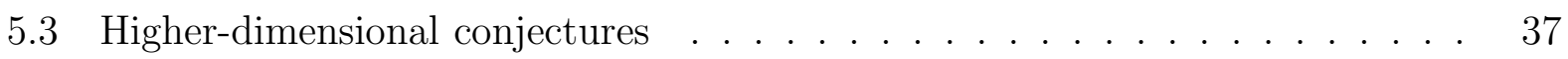

6 Conclusions $\quad 37$

\begin{tabular}{ll|l}
7 & Acknowledgements & 38
\end{tabular}

\begin{tabular}{l|l|} 
A Topological classification of extensions & 38 \\
\hline
\end{tabular} 
References

40 


\section{Introduction}

The recent paper [1] proposed a more general class of symmetries that should be studied in quantum field theories: in addition to actions of ordinary groups, it proposed that we should also consider 'groups' of gauge fields and higher-form analogues. For example, Wilson lines can act as charged objects under such symmetries. By using various defects, the paper [1] described new characterizations of gauge theory phases.

Now, one can ask to what extent it is natural for $n$-forms as above to form a group. In particular, because of gauge symmetries, the group multiplication will not be associative in general, unless perhaps one restricts to suitable equivalence classes, which does not seem natural in general. A more natural understanding of such symmetries is in terms of weaker structures known as 2-groups and higher groups, in which associativity is weakened to hold only up to isomorphisms.

There are more 2-groups and higher groups than merely, 'groups' of gauge fields and higher-form tensor potentials (connections on bundles and gerbes), and in this paper we will give examples of actions of such more general higher groups in quantum field theory and string theory. We will also propose an understanding of certain anomalies as transmutations of symmetry groups of classical theories into higher group actions on quantum theories.

To be clear, proposals for roles of 2-groups in physics have appeared previously in a number of papers, in e.g. [2-5], in discussions of the String group (see e.g. [6] 9]), in the Yetter model (see e.g. [10,11]), and in lattice gauge theories (see e.g. [12, 13]), to name a few examples. Additional categorical generalizations of (orbifold) groups, via an application of defects to generalize the ordinary orbifold construction, are discussed in [14-19]. See also [20] for related ideas and applications of discrete gauge theories and group cohomology in condensed matter physics. The purpose of this paper is to merely to link the recent work [1] to other work on 2-groups, to review a few highlights, and to provide a few hopefully new results, proposals, and applications.

We begin in section 2 with a brief introduction to 2-groups and higher groups, omitting mathematical details for the sake of readability. In section 3 we describe several examples of quantum field theories admitting higher group symmetries. We begin with a brief review of the gauge theory phase analysis of [1], then describe symmetries of gauge theories with massless matter that is invariant under a finite subgroup of the gauge group. (Such theories have been studied in a number of papers, and in two dimensions naturally decompose into disjoint unions of theories, as is reviewed in that section.) We also review how boundary structures in Dijkgraaf-Witten theory and also WZW models define further examples of theories admitting higher group symmetries, albeit more complicated higher groups than just $p$-form symmetries. Both those examples of higher group symmetries are discussed elsewhere in the literature, but are reviewed here as they play a role later. At the end of that section, we also speculate on how current algebras in higher dimensions may define further examples 
of higher group symmetries, and also mention some other potential applications. In section 4 we briefly discuss cosmological defects in the context of higher group symmetries, as well as 'generalized moduli spaces' (technically, stacks), defined here as 'spaces' which admit actions of higher groups. Finally, in section 5 we conjecture that some anomalies might be interpreted as promoting classical ordinary-group symmetries to quantum higher-group symmetries.

One of the ideas that drives this paper is that when the action of an ordinary group $G$ is broken - in mathematics, by trying to lift $G$ to a bundle that does not admit a $G$ action, say, or in physics, via anomalies - there is often a larger or higher group that will act instead. We will encounter various manifestations of this principle in several contexts.

An idea that has often been repeated is that one should look for fundamental symmetries to better understand string theory (see for example [21] for one well-known example). The philosophy espoused by this paper and others mentioned above is that perhaps instead of only looking for ordinary group symmetries, we should also look for higher group symmetries.

\section{Overview of 2-groups and higher groups}

In this section, to make this paper self-contained, we will outline definitions and examples of 2-groups and higher groups, as these ideas are not widely known in the physics community. We will begin with 2-groups (see e.g. [6, 8, 22, 26] for discussions and introductions to 2groups).

Briefly, given objects $x, y, z$ in a 2-group, instead of demanding that multiplication be strictly associative

$$
(x \cdot y) \cdot z=x \cdot(y \cdot z),
$$

we instead demand that the two sides be merely isomorphic, related by isomorphisms

$$
\alpha(x, y, z):(x \cdot y) \cdot z \stackrel{\sim}{\longrightarrow} x \cdot(y \cdot z) .
$$

Similarly, the identity axioms

$$
1 \cdot x=x, \quad x \cdot 1=x
$$

are also replaced by isomorphisms

$$
\ell_{x}: 1 \cdot x \stackrel{\sim}{\longrightarrow} x, \quad r_{x}: x \cdot 1 \stackrel{\sim}{\longrightarrow} x
$$

and identity relations

$$
x \cdot x^{-1}=1, \quad x^{-1} \cdot x=1
$$


are also replaced by isomorphisms. These isomorphisms must satisfy relations of the form (see e.g. [24])

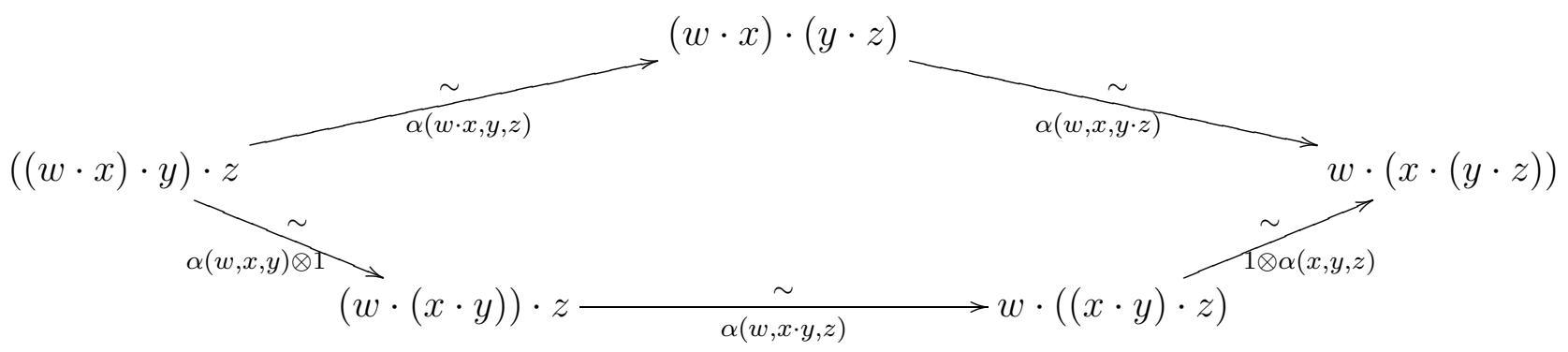

and

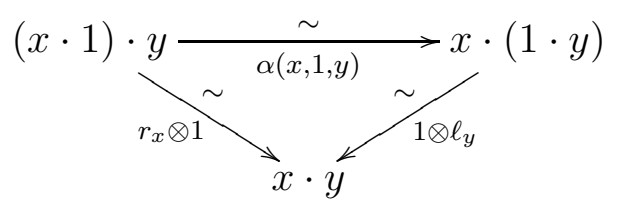

There are technical distinctions between various descriptions of 2-groups, which will not be relevant for our purposes here (see instead e.g. [24]).

The description above suggests that it may be possible to construct 2-groups from a given group by weakening associativity by an automorphism defined by an element of degree 3 group cohomology, and in fact, this is the case, see e.g. [24][section 8.3], [26, 27]: for a given group $G$, group cohomology $H^{3}(G, U(1))$ (with trivial action on the coefficients) defines a corresponding 2-group. Briefly, the basic idea is that a 3-cocycle $\alpha(g, h, k)$ defines an isomorphism

$$
(g \cdot h) \cdot k \stackrel{\sim}{\longrightarrow} g \cdot(h \cdot k)
$$

and as the cocycle is closed in group cohomology, the isomorphisms so defined satisfy the pentagon identity:

$$
\alpha(g, h, k) \alpha(g, h k, \ell) \alpha(h, k, \ell)=\alpha(g h, k, \ell) \alpha(g, h, k \ell) .
$$

We shall return to this example shortly.

Let us next discuss 2-groups appearing in discussion of "1-form symmetries." For $G$ abelian, we can define [28] the 2-groups $\mathbf{B} G, \mathbf{B} G_{\text {conn }}$, and $\mathbf{B}_{b} G_{\text {conn }}$, which we will define over the next several paragraphs. The first 2-group $\mathbf{B} G$ assigni 1 to any manifold the category of principal $G$ bundles. There exists a tensor product that tensors any two $G$ bundles to produce another $G$ bundle 2 , and the resulting operation defines a multiplication that is associative up to higher homotopies, as above.

\footnotetext{
${ }^{1}$ Technically, in our conventions we will take $\mathbf{B} G$ to be the stack [point/ $G$ ]. More generally, classifying stacks and classifying spaces naturally admit the structure of higher groups. We will largely avoid using the language of stacks in this paper so as to make it more accessible, aside from technical footnotes of this form.

${ }^{2}$ Technically, we should describe this in terms of tensor products of torsors [29] [section 5.1], [2], but for the purposes of this paper, we shall be content with looser language.
} 
The 2-group $\mathrm{B} G_{\text {conn }}$ is the analogous classifying object for principal $G$ bundles with connection, which tensors bundles and adds the connections. (If $G$ is finite, then as there is no nontrivial connection on a principal $G$-bundle, $\mathbf{B} G_{\text {conn }}=\mathbf{B} G$.) This 2-group appeared implicitly in [1]. Since the isomorphisms must preserve connections and so are more restricted than the isomorphisms in $\mathbf{B} G$ above, one might worry that the resulting 2-groups would all be equivalent to ordinary groups. However, this is not the case. For example, discrete torsion implicitly provides examples of 2-groups of this form which are not equivalent to ordinary groups. As discussed in e.g. [30], discrete torsion arises from choices of equivariant structures on $B$ fields - one combines the action of the orbifold group $\Gamma$ on the underlying space (here, a point) with gauge transformations of the $B$ field. The gauge transformations, themselves principal $U(1)$ bundles with connection, are required to obey the group law up to isomorphism - in essence, a 2-group structure - and those isomorphisms are encoded in elements of $H^{2}(\Gamma, U(1))$, as connection-preserving gauge transformations of a principal $U(1)$ bundle.

As 2-groups, $\mathbf{B} G$ and $\mathbf{B} G_{\text {conn }}$ are not equivalent to one another [27,28]. Now, that said, to classify 2-groups topologically, we will sometimes replace 2-groups by their 'geometric realization.' Ultimately because the space of connections on a fixed bundle is contractible, the geometric realization of $\mathbf{B} G$ is homotopic to that of $\mathbf{B} G_{\text {conn }}$, despite the fact that the 2 -groups are not equivalent [31]. In both cases, the geometric realization is homotopic to the ordinary classifying space for $G$, which we will denote as $B G$ (with a non-boldface $B$ ), to distinguish it from the 2-groups above. (See for example [32] for a basic discussion of the classifying space, and appendix $\AA$ for references on homotopies of geometric realizations.)

Now, if one lifts to a loop space, some of this structure simplifies. For example, $G$-gerbes over a space $X$ become principal $G$-bundles on the loop space $L X$. In particular, a Wilson line is effectively a function on $L X$, and so obeys an ordinary group law. Thus, if one is working with these structures exclusively through Wilson lines, then at least to some extent it is entirely reasonable to speak about them as ordinary groups (involving Wilson lines) rather than higher groups, as in fact was done in [1].

Finally, let us discuss $\mathbf{B}_{b} G_{\text {conn }}$. This is the analogue of $\mathbf{B} G_{\text {conn }}$ for flat bundles. In other words, $\mathbf{B}_{b} G_{\text {conn }}$ assigns the category of flat $G$ bundles with connection. If $G$ is finite, then for trivial reasons

$$
\mathbf{B}_{b} G_{\text {conn }}=\mathbf{B} G=\mathbf{B} G_{\text {conn }} .
$$

Next let us turn to further examples of 2-groups, which cannot be understood as simply as "1-form symmetries." In particular, we will next consider examples of 2-groups formed as extensions, as described in e.g. [6]. For $G$ a group, there are several 2-group extensions $\tilde{G}$ given by

$$
1 \longrightarrow \mathrm{B} U(1) \longrightarrow \tilde{G}_{1} \longrightarrow G \longrightarrow 1,
$$

\footnotetext{
${ }^{3}$ Further discussion of the relation between these extensions can be found in e.g. [33].
} 


$$
\begin{gathered}
1 \longrightarrow \mathrm{B} U(1)_{\text {conn }} \longrightarrow \tilde{G}_{c} \longrightarrow G \longrightarrow 1, \\
1 \longrightarrow B U(1) \longrightarrow \tilde{G}_{0} \longrightarrow G \longrightarrow 1,
\end{gathered}
$$

differing by whether one extends by a stack $\mathbf{B} U(1), \mathbf{B} U(1)_{\text {conn }}$, or a classifying space $B U(1)$.

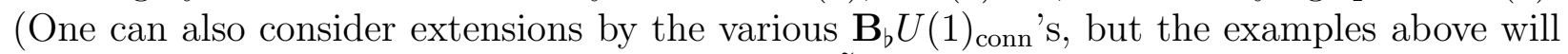
suffice for this paper.) Extensions of the form $\tilde{G}_{1}$ are discussed in [6]. In particular, this extension can be understood as a $U(1)$-gerbe over $G$, with suitable multiplicative structure. For $G$ finite, these are precisely the 2-gerbes discussed above which are classified by group cohomology $H^{3}(G, U(1))$.

Let us now suppose instead that $G$ is simple and simply-connected; in this case, extensions of this form above are classified by $H^{3}(G, \mathbb{Z}$ ) (see appendix $\mathbb{A}$ ). (Each type of extension is classified in the same way, as discussed in appendix A.) Now, for $G$ simple and simplyconnected, $H^{3}(G, \mathbb{Z})=\mathbb{Z}$, and so possible extensions are classified by an integer. The reader may find this structure reminiscent of WZW models, and indeed there is a close connection: the underlying $U(1)$-gerbe appearing in WZW models, for which the Wess-Zumino term acts as a coupling to the $B$ field, is precisely the 2 -group $\tilde{G}_{1}$ above (with suitable multiplication), and the integer classifying the extension is the same as the level of the WZW model. We will return to this connection later.

So far this discussion has been rather abstract. Let us now try to make it more concrete by discussing more explicitly the multiplication on a 2 -group $\tilde{G}$, built as an extension of (simple, simply-connected) $G$ by some version of $\mathbf{B} U(1)$. This product is more easily described on the loop space of $G$, or rather a minor variation of the loop space. (The resulting description will also implicitly encode a version of Wilson lines for 2-groups defined as extensions $\tilde{G}$ of the form above.)

First, let us describe elements of $\tilde{G}$. Following [29][section 6.4], we can describe them as certain equivalence classes of pairs $(\sigma, z)$, where $\sigma: D^{2} \rightarrow G$ for $D^{2}$ a two-dimensional disk (corresponding to a filled-in loop) and $z \in U(1)$ (corresponding to an ordinary $U(1)$ Wilson line). Then the product of two elements of $\tilde{G}$ (over this analogue of the loop space) is defined by

$$
\left(\sigma_{1}, z_{1}\right) \cdot\left(\sigma_{2}, z_{2}\right)=\left(\sigma_{1} \sigma_{2}, z_{1} z_{2} \exp \left(-2 \pi i \int_{D^{2}}\left(\sigma_{1} \times \sigma_{2}\right)^{*} \omega\right)\right),
$$

where $\omega$ is a 2-form on $G \times G$, defined as follows. If $\nu$ is the a multiple of the canonical 3form on $G$ (associated with the Wess-Zumino term in WZW models, and encoding the level), $p_{1,2}: G \times G \rightarrow G$ are projection maps, and $m: G \times G \rightarrow G$ the ordinary multiplication in $G$, then

$$
p_{1}^{*} \nu+p_{2}^{*} \nu-m^{*} \nu=d \omega .
$$

(Intuitively, we can think of $\sigma$ as a semiclassical state of the string in a WZW model, and the phase acquired in multiplication as the integral of the Wess-Zumino term over the path swept out by the change in region.) As this is defined over (a version of) the loop space, rather than over $G$ itself, this is an honest group structure: associativity holds on the nose, 
rather than up to cocycles. In more detail, associativity of the multiplication follows from the fact that for any $\sigma_{1,2,3}$,

$$
\int\left(\sigma_{2} \times \sigma_{3}\right)^{*} \omega+\int\left(\sigma_{1} \times \sigma_{2} \sigma_{3}\right)^{*} \omega=\int\left(\sigma_{1} \times \sigma_{2}\right)^{*} \omega+\int\left(\sigma_{1} \sigma_{2} \times \sigma_{3}\right)^{*} \omega
$$

as follows from the fact that these four terms correspond schematically to the four faces of a tetrahedron, as illustrated schematically below:

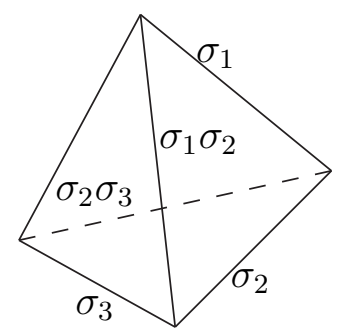

In passing, we should mention that there is a related story for Lie algebroids. Given a Lie algebroid $\bar{Q}$ associated to some bundle $P$, the obstruction to lifting to a nontrivial Courant algebroid $Q$ determined by an extension of $\bar{Q}$ by $\Omega^{1}$ :

$$
0 \longrightarrow \Omega^{1} \longrightarrow Q \longrightarrow \bar{Q} \longrightarrow 0
$$

is given by the first Pontryagin class $p_{1}(P)$ [34]. The relevance of this construction to the Green-Schwarz condition and associated anomalies in heterotic strings has been discussed in e.g. [35 37]; see also [38] for a discussion of the Green-Schwarz anomaly in present language.

In principal, $n$-groups for $n>2$ can be defined similarly to 2 -groups, by breaking the relations above to only hold up to higher levels of isomorphisms, which themselves obey higher identities. For example, to get a 3-group, one would replace the identity

$$
\alpha(w, x, y \cdot z) \circ \alpha(w \cdot x, y, z)=(1 \otimes \alpha(x, y, z)) \circ \alpha(w, x \cdot y, z) \circ(\alpha(w, x, y) \otimes 1)
$$

with isomorphisms

$\beta(w, x, y, z): \alpha(w, x, y \cdot z) \circ \alpha(w \cdot x, y, z) \stackrel{\sim}{\longrightarrow}(1 \otimes \alpha(x, y, z)) \circ \alpha(w, x \cdot y, z) \circ(\alpha(w, x, y) \otimes 1)$

which themselves obey higher identities.

The theory of $n$-groups seems to be less well developed than that of 2 -groups; we refer the reader to e.g. [39 41] for a few details. Briefly, the complications in making sense of $n$-groups are tied into the complications of understanding higher categories, and in modern language, are perhaps best understood by working with $\infty$-categories (and hence $\infty$-groups). We will not need that level of technology, but we will occasionally make conjectures based 
formally on $n$-groups, so we will describe a few examples we shall use in this paper, and for technical definitions we refer interested readers to e.g. [41.

In that spirit, for $G$ abelian, we will define $[28] \mathbf{B}^{q} G$ to associate to any manifold the $G$ $(q-1)$-gerbes on that manifolds, $\mathbf{B}^{q} G_{\text {conn }}$ the $G(q-1)$-gerbes with connection, and $\mathbf{B}_{b}^{q} G_{\text {conn }}$ the flat $G(q-1)$-gerbes with connection. If $G$ is finite, then for trivial reasons

$$
\mathbf{B}_{b}^{q} G_{\text {conn }}=\mathbf{B}^{q} G=\mathbf{B}^{q} G_{\text {conn }} .
$$

To make our notation uniform, we shall define $\mathbf{B}^{0} G$ to be the ordinary group of smooth maps from the manifold into $G$, and $\mathbf{B}_{b}^{0} G_{\text {conn }}$ the ordinary group of constant maps into $G$. (The classifying space for the former is $G$; for the latter, $K(G, 0)$.) We then recognize that in more typical symmetry discussions in $\mathrm{QFT}, \mathbf{B}_{b}^{0} G_{\text {conn }}$ defines a global symmetry group, and $\mathbf{B}^{0} G$ a local symmetry group. Analogous parallels hold for $q>0$.

Analogues of the extension construction above exist for higher groups [28, 41, 42]. For example, one can consider extensions of the form

$$
\begin{gathered}
1 \longrightarrow \mathbf{B}^{k} U(1) \longrightarrow \tilde{G}_{1, k} \longrightarrow G \longrightarrow 1, \\
1 \longrightarrow \mathbf{B}^{k} U(1)_{\mathrm{conn}} \longrightarrow \tilde{G}_{c, k} \longrightarrow G \longrightarrow 1, \\
1 \longrightarrow B^{k} U(1) \longrightarrow \tilde{G}_{0, k} \longrightarrow G \longrightarrow 1,
\end{gathered}
$$

differing by whether one extends by a higher stack $\mathbf{B}^{k} U(1), \mathbf{B}^{k} U(1)_{\text {conn }}$ or a space $B^{k} U(1)$. As outlined in appendix $\mathbb{\mathrm { A }}$, these are classified by $H^{k+2}(G, \mathbb{Z})$ for Lie groups $G$ of nonzero dimension, and group cohomology $H^{k+2}(G, U(1))$ for finite $G$.

\section{Examples of higher group symmetries in QFT}

In this section we list some examples of higher group symmetries in quantum field theories.

\subsection{Review of $q$-form symmetries in gauge theories}

To make this paper self-contained, let us begin with a very brief outline of the highlights of some of the examples of global $q$-form symmetries (special cases of higher group symmetries) discussed in [1].

\footnotetext{
${ }^{4}$ Readers familiar with the bar construction of classifying spaces will recognize the deliberate notational parallel.
} 
Reference [1] gives a number of examples 5 of global $q$-form symmetries involving shifts of an existing gauge field. The prototype for many of their examples was a four-dimensional $U(1)$ gauge theory on some general four-manifold. This admits an (electric) action 6 of $\mathbf{B}_{b} U(1)_{\text {conn }}$ defined by shifting the gauge field $A$ by a flat $U(1)$ connection $A_{0}$, as $A \mapsto A+A_{0}$, and tensoring the gauge bundle by the corresponding flat $U(1)$ bundle. There is also a dual magnetic action of $\mathbf{B}_{b} U(1)_{\text {conn }}$, defined by shifting the magnetic potential in the same fashion.

Note that under the action above, Wilson lines are charged, picking up the holonomy of the element of $\mathbf{B}_{b} U(1)_{\text {conn }}$ around the path:

$$
\exp \left(\int A \cdot d s\right) \mapsto \exp \left(\int A \cdot d s\right) \exp \left(\int A_{0} \cdot d s\right)
$$

Mathematically, this can be understood in terms of passing to the (based) loop space: line bundles on a given space become functions on loop space, and $\mathbf{B}_{b} U(1)_{\text {conn }}$ becomes $B_{b}^{0} U(1)_{\text {conn }}$, the group of constant $U(1)$ elements, on the loop space, so that overall this oneform symmetry reduces to an ordinary (zero-form) group symmetry on the loop space. (That said, Wilson lines themselves can at least sometimes define a 2-group, see e.g. [47][section 3].)

If a Wilson line has a nonzero vev in the limit of large 7 loop sizes, then the one-form $\mathbf{B}_{b} U(1)_{\text {conn }}$ symmetry said to be spontaneously broken. Conversely, if one knows that a symmetry of this form is unbroken, one can argue that Wilson loop vevs in large loop limits must vanish, a selection rule on Wilson lines discussed in [1].

Reference [1] observes that the gauge field $A_{\mu}$ is naturally understood as the Goldstone boson associated with that spontaneously broken symmetry, as it undergoes a translation under the symmetry group action.

To close the intellectual loop, it would be helpful to see explicitly that the state space of an abelian gauge theory has the structure of a 2-vector-space in a representation of $\mathbf{B}_{b} U(1)_{\text {conn }}$. At a very formal level, such a description was proposed in [48], which we shall outline here8. (Readers unfamiliar with stacks may wish to skip the rest of this paragraph.) Briefly, the idea is to think of states in abelian gauge theory on a manifold $X$ (expanding about an arbitrary principal $U(1)$ bundle with connection) as defined by wavefunctions, defined on a subset of $\left[X, \mathbf{B} U(1)_{\text {conn }}\right]$ satisfying a polarization condition. Using "crit" to label the pertinent subset,

\footnotetext{
${ }^{5}$ See also [43 46] for related ideas.

6 In more formal language [28, we could describe the gauge field $A$ and corresponding bundle via a

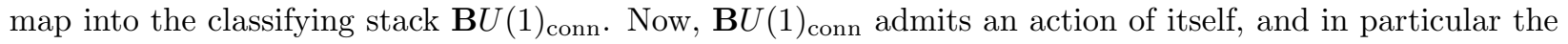
substack $\mathbf{B}_{b} U(1)_{\text {conn }}$, which is the action being described above.

7 The reader might well observe that any nonzero vev of an operator that transforms under a symmetry defines a spontaneous symmetry breaking. Here, however, we intend to refer to the phase visible in the IR, for which the limit of large loop size is relevant. In any event, we follow the convention set in 1 .

${ }^{8}$ We thank U. Schreiber for outlining his argument to us, which we repeat here.
} 
the wavefunctions are elements of the 2-vector-space

$$
\left[\left[X, \mathbf{B} U(1)_{\text {conn }}\right]_{\text {crit }}, \mathbb{C}\right] \text {, }
$$

which naturally admits an action of $\mathbf{B}_{b} U(1)_{\text {conn }}$ [28]. More globally, we are under the general impression that the representation theory of higher groups is still under development, so in the rest of this paper we will occasionally make physics-based conjectures regarding representations, but for the most part we will not be able to make strong statements of results concerning representations.

Some related examples discussed in [1] include:

- A four-dimensional $G$ gauge theory admits a global action of $\mathbf{B}_{b} Z(G)_{\text {conn }}$ defined by shifting the gauge field by a flat $Z(G)$ connection, and similarly tensoring the gauge bundle by the corresponding flat $Z(G)$-bundle.

- A theory with a dynamical $q$-form potential admits a global action of $\mathbf{B}_{b}^{q} U(1)$ conn given by shifting the $q$-form potential by a flat $q$-form, and tensoring the $q$-gerbe by the corresponding flat $q$-gerbe. (See also [49] and references therein for related examples.)

In all these cases, various defect:10 play a role analogous to charged particles for ordinary symmetries.

In any event, the paper [1] focused on analyzing gauge theory phases using the ideas above, whereas in this paper we will look in different directions.

\subsection{Gauge theories with subgroup-invariant massless matter}

In this section we shall study gauge theories in which a finite subgroup of the center of the gauge group acts trivially on massless matter, and related orbifolds, as further examples of theories with generalized global symmetries.

As a warm-up, consider the two-dimensional supersymmetric $\mathbb{C} \mathbb{P}^{n}$ model, consisting of a $U(1)$ gauge theory with $n+1$ charged massless chiral multiplets, but rather than giving all the chiral multiplets charge 1 as in the usual construction, instead give them all charge $k$. Perturbatively, this would appear to be the same as the ordinary $\mathbb{C P}^{n}$ model, in which all matter fields have charge 1 , but in fact these cases can be distinguished:

- One way to distinguish these theories is by adding massive fields of charge \pm 1 to the theory with massless charge $k$ matter. The existence of this massive matter can still

\footnotetext{
$9 Z(G)$ denotes the center of $G$.

10 See for example [50] for a discussion of defects in topological field theories.
} 
be detected even below the cutoff scale, via the periodicity of the theta angle, which acts as an electric field in two dimensions. To see this, simply build a capacitor in the two-dimensional theory; as one increases its size, one can excite arbitarily massive matter, and so the theta angle periodicity provides a test for charged matter beyond the renormalization cutoff scale. (For more information, see for example [51 53], where this model was originally discussed.)

- In principle, one could use defects to distinguish these theories. For example, one could specify that the theory contains Wilson lines in the charge 1 representation. Such Wilson lines would not be well-defined after dividing the charges by $k$, and so specifying their existence would distinguish these theories. Such structures were discussed in fourdimensional theories in the context of discrete theta angles in [54,55].

- In principle, the global structure of the gauge group could also be detected via gauge transformations on non-simply-connected spacetimes. Put another way, if the spacetime is topologically nontrivial, then to specify matter fields, one must specify a bundle to which the matter couples, not just a representation. The specification of the bundle eliminates any ambiguities, and as different bundles lead to different zero modes and different anomalies, one also sees distinct physics. This is also discussed in for example [51,53].

At low energies, below the scale of any massive minimally charged matter, this theory has a $\mathbf{B} \mathbb{Z}_{k}$ symmetry, acting by translations of the $U(1)$ gauge field in a $\mathbb{Z}_{k}$ subgroup, as this leaves the action invariant. (This same group may act nontrivially on defects that distinguish the charge $k$ and charge 1 massless matter cases; however, a nontrivial action on such operator vevs should be interpreted in terms of spontaneous symmetry breaking, rather than explicit symmetry breaking.) This $\mathbf{B} \mathbb{Z}_{k}$ action is of the same form as discussed in the last section: the gauge bundle is 'tensored' with a $\mathbb{Z}_{k}$ bundle, for example. However, at higher energies, this $\mathbf{B} \mathbb{Z}_{k}$ symmetry is explicitly broken by any massive minimally charged matter, as the action is no longer invariant.

We could also discuss the theory without massive minimally charged matter or defects fixing the global structure of the gauge group. This theory, in which the charges of all matter fields are multiples of $k$, naively appears to also have a $\mathbf{B} \mathbb{Z}_{k}$ symmetry, but this symmetry is an artifact of the charge scaling, and so has no physical significance.

Another set of examples involves orbifolds by finite groups that do not act effectively on the target space. A simple example, discussed extensively in e.g. [52], is the orbifold $\left[X / D_{4}\right]$, where $D_{4}$ is the eight-element group described as an extension

$$
1 \longrightarrow \mathbb{Z}_{2} \longrightarrow D_{4} \longrightarrow \mathbb{Z}_{2} \times \mathbb{Z}_{2} \longrightarrow 0
$$

and where $D_{4}$ acts on $X$ by first projecting to $\mathbb{Z}_{2} \times \mathbb{Z}_{2}$, so that the remaining $\mathbb{Z}_{2}$ acts trivially on $X$. By computing partition functions, it is simple to demonstrate that this theory is not 
the same as the $\left[X / \mathbb{Z}_{2} \times \mathbb{Z}_{2}\right]$ orbifold - both theories admit modular-invariant partition functions, but those modular-invariant partition functions are different. In particular, if $H$ is a subgroup of the orbifold group that acts completely trivially on the space, then the theory admits a $\mathbf{B} H$ action. In this case, the $\left[X / D_{4}\right]$ orbifold admits a $\mathbf{B} \mathbb{Z}_{2}$ action.

It is straightforward to see the $\mathbf{B} \mathbb{Z}_{k}$ actions in orbifold examples, but for completeness, we shall work through two examples here.

- First, consider an orbifold $\left[X / \mathbb{Z}_{k}\right]$ where all of the $\mathbb{Z}_{k}$ acts trivially on $X$. In other words, for all $x \in X$ and all $g \in \mathbb{Z}_{k}, g \cdot x=x$. The one-loop partition function of this theory is involves a sum over principal $\mathbb{Z}_{k}$ bundles, of the form

$$
Z=\frac{1}{\left|\mathbb{Z}_{k}\right|} \sum_{g, h \in \mathbb{Z}_{k}} Z(X)=\left|\mathbb{Z}_{k}\right| Z(X) .
$$

(A principal $\mathbb{Z}_{k}$ bundle on $T^{2}$ is specified by a commuting pair of elements of $\mathbb{Z}_{k}$, hence the sum over $g, h \in \mathbb{Z}_{k}$.) The action of $\mathbf{B} \mathbb{Z}_{k}$ is merely to rotate the bundles amongst themselves. For example, given another bundle defined by a (commuting) pair $\left(g^{\prime}, h^{\prime}\right)$, the action on the bundle defined by the pair $(g, h)$ is merely

$$
(g, h) \mapsto\left(g^{\prime} g, h^{\prime} h\right) .
$$

- A somewhat less trivial example is provided by the $\left[X / D_{4}\right]$ example given above. This theory admits a $\mathbf{B} \mathbb{Z}_{2}$ action, which we can see in the one-loop partition function as follows. Write the one-loop partition function as

$$
Z=\frac{1}{\left|D_{4}\right|} \sum_{g, h \in D_{4}, g h=h g} Z_{g, h}(X),
$$

where the sum is over commuting pairs of elements of $D_{4}$ (i.e. principal $D_{4}$ bundles on $T^{2}$ ), and $Z_{g, h}(X)$ denotes the partition function with boundary conditions determined by $g, h$. Now, a $\mathbb{Z}_{2}$ bundle is determined by a pair of elements of $\mathbb{Z}_{2} \subset D_{4}$, whose elements we will denote $\{1, z\}$, where $z^{2}=1$. Thus, a general $\mathbb{Z}_{2}$ bundle is given by a pair $\left(z^{m}, z^{n}\right)$ for integer $m, n$. Such a bundle will act on a $D_{4}$ bundle $(g, h)$ as

$$
(g, h) \mapsto\left(g z^{m}, h z^{n}\right)
$$

Since the $\mathbb{Z}_{2}$ acts trivially on $X$,

$$
Z_{g z^{m}, h z^{n}}=Z_{g, h},
$$

and so the partition function is preserved. 
More generally, for any $G$ orbifold of a space $X$, if a subgroup $H$ acts trivially on $X$, then in the same fashion as above, $\mathbf{B} H$ defines a symmetry of the theory, as can be seen in rotations of the $G$ bundles by $H$ subbundles.

Four-dimensional analogues were discussed in [56]. Two examples of this form are as follows:

- A $U(1)$ gauge theory with $N$ matter fields of charge $+k$ and $N$ matter fields of charge $-k$.

- An $S U(2)$ gauge theory with adjoints.

At low energies, generically along the Higgs branch, the second reduces to a $U(1)$ gauge theory with matter of charge divisible by 2 , i.e. an example of the former theory. As in two dimensions, these four-dimensional theories can be distinguished from minimally-charged matter theories in several ways:

- One option is to add massive minimally-charged matter to the theory, as in two dimensions. The presence of such matter can no longer be sensed by the theta angle periodicity, as the theta angle no longer acts as an electric field, but instead in a theory coupled to gravity, one can use Reissner-Nordstrom black holes to similar effect, as discussed in [56].

- Another option is to specify a set of defects in the theory which are well-defined only for certain global gauge groups. This was the strategy followed in [54,55] to distinguish $S U(2)$ from $S O(3)$ theories, for example.

- Finally, as before, if the spacetime is topologically nontrivial, then a unique specification of the matter will have the same ffect.

In two dimensions, gauge theories in which a subgroup of the gauge group acts trivially on massless matter are equivalent to theories with restrictions on nonperturbative sectors. In four dimensions, the analogue is more complicated. This matter is discussed in greater detail in [56, 58]. These theories can also be understood, in different language still, in terms of QFT's coupled to TFT's, though we shall not use that language here.

There are many other related examples, in which massless matter is invariant under a subgroup $G$ of the center of the gauge group, and these examples all have a $\mathbf{B} G$ symmetry at low energies.

These theories are sometimes known as gerbe theories, because they are typically sigma models on gerbes as discussed in, for example, [53,57]. Very briefly, for those readers who are curious, a sigma model on a (Deligne-Mumford) stack is defined by first picking a presentation 
of the stack as a global quotient $[X / G]$ of some ordinary space $X$ by some group $G$, which need not be finite and need not act effectively (but whose stabilizers on $X$ are finite). The 'sigma model on the stack' is then a $G$-gauged sigma model on $X$, or rather, its universality class. If a subgroup $H$ of $G$ acts trivially on $X$, then the stack is known technically as an $H$ gerbe, and the theory is a sigma model on a gerbe.

We have already seen how theories of this form admit $\mathbf{B} H$ actions - by rotating principal $G$ bundles by $H$ subbundles, which (as $H$ acts trivially) leaves the theory invariant. (This is true for both gauge theories and orbifolds, and we have seen examples of each.) As sigma models on gerbes, we can view these $\mathbf{B} H$ structures another way. An $H$-gerbe (for $H$ finite) over a space is, mathematically, the total space of a $\mathbf{B} H$ bundle. As such, $\mathbf{B} H$ acts on the fibers of the bundle, and so acts on the gerbe and, physically, on the sigma model.

The same language gives another perspective on sigma models on gerbes. As has been discussed elsewhere (see e.g. [56]), in two dimensions these are equivalent to sigma models with restrictions on nonperturbative sectors. One way to think about the origin of those restrictions is as a requirement that the sigma model maps preserve the $\mathbf{B} H$ invariances.

The Higgs moduli spaces of these theories also have gerbe structures, and so admit actions of higher groups. We will explore this point in greater detail in section 4.2, where such moduli spaces will be described as 'generalized moduli spaces.'

So far we have discussed some basic examples. It has been shown that dualities break the $\mathbf{B} G$ symmetries discussed in this section. Specifically, it has now been established that these two-dimensional theories 'decompose' (via a form of T-duality) into disjoint unions of simpler theories, without these $\mathbf{B} G$ symmetries, which solves a technical issue with cluster decomposition, see e.g. [57] for a discussion of decomposition in two-dimensional nonlinear sigma models, [58] for a discussion of decomposition in two-dimensional nonabelian gauge theories, and [59] for a heterotic version. This decomposition makes predictions for GromovWitten invariants, which have been checked rigorously (see e.g. [60 65]), and also plays a role in understanding phases of certain GLSMs [57, 66 68] (see [69] for a more complete list of references and reviews). It would be interesting to understand if there are analogues of decomposition for any notion of $\mathbf{B} G$ gauge theories in some dimension. In any event, reference [56] contains a study of how these gerbe structures over moduli spaces vary under dualities.

\subsection{Boundary structures in Dijkgraaf-Witten theory}

Recall that for finite $G$, an element of group cohomology $H^{3}(G, U(1)$ ) (with trivial action on the coefficients) defines a 2 -group $\tilde{G}$ as the extension

$$
1 \longrightarrow \mathrm{B} U(1) \longrightarrow \tilde{G} \longrightarrow G \longrightarrow 1 \text {. }
$$


In this section, we shall review arguments that this 2-group $\tilde{G}$ acts on the boundaries in three-dimensional Dijkgraaf-Witten theories [70] defined by the corresponding element of $H^{3}(G, U(1))$. (We emphasize that the observations of this subsection are not original to us, but instead are reviewed for completeness and later use in this paper. See instead e.g. [41][p. 757] for references.)

First, consider the special case of a trivial $G$ gauge theory in two dimensions, for $G$ finite a $G$-orbifold - with discrete torsion. Discrete torsion is defined by an element of $H^{2}(G, U(1))$ (with trivial action on the coefficients), and elements of that same group cohomology group determine a central extension $\tilde{G}$ :

$$
1 \longrightarrow U(1) \longrightarrow \tilde{G} \longrightarrow G \longrightarrow 1 \text {. }
$$

As was argued in $[30,71-73]$, the extension $\tilde{G}$ acts on the Chan-Paton factors and D-branes. The extension $\tilde{G}$ is still an ordinary group, but this example provides a prototype for the 2group action that will appear on boundaries of three-dimensional Dijkgraaf-Witten theories.

Abstractly, to define the two-dimensional $G$ gauge theory in the presence of the boundary, one must express how the group $G$ acts on the Chan-Paton factors as encoded in a Wilson line along the boundary (or equivalently a bundle $L$ with connection). If the $G$ action lifts honestly, meaning that there are isomorphisms $\psi_{g}: g^{*} L \rightarrow L$ such that the $\psi_{g}$ 's obey the group law, then $L$ is said to admit a $G$-equivariant structure. In general, however, the group law might not be obeyed, meaning the lift of $G$ to the boundary theory is obstructed. Such obstructions are encoded in element:11 of $H^{2}(G, U(1))$. In such a case, although the action of $G$ on the boundary theory is obstructed, there is an action of $\tilde{G}$ (the lift associated with the same element of $\left.H^{2}(G, U(1))\right)$ on the boundary theory: the boundary theory is always $\tilde{G}$-equivariant, for the extension determined by the obstruction to $G$-equivariance.

There is a closely analogous story in three-dimensional Dijkgraaf-Witten theories [70]. To help clarify the discussion, we shall assume that the three-dimensional finite $G$ gauge theory includes a trivial action on a nonlinear sigma model on some space $M$. (It will become clear that $M$ is irrelevant.) Then, a boundary in the theory should have an action which includes a term of the form

$$
\int \phi^{*} B
$$

for some two-form potential $B$, and map $\phi$ from the three-dimensional spacetime into $M$. Assuming that the boundary of the boundary is empty, this theory has a natural action of the 2-group $\mathbf{B} U(1)_{\text {conn }}$, which acts on $B$ by gauge transformations of the familiar form $B \mapsto B+d \Lambda$. However, this is only part of the 2-group that acts on the boundary of this theory.

Since the bulk contains a $G$ gauge theory, to fully describe the theory, we must describe how $G$ acts on the boundary. In general, $G$ can be combined with gauge transformations

\footnotetext{
${ }^{11}$ In this context, $H^{2}(G, U(1))$ is known as Mumford's group of $L$ or the theta group of $L$ [74].
} 


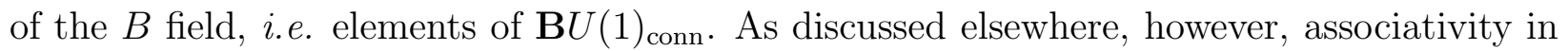

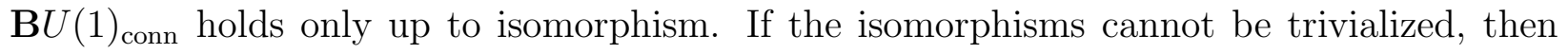
we do not have any sort of homomorphism $G \rightarrow \mathbf{B} U(1)_{\text {conn }}$, but rather a more complicated structure comes into play.

We can link the breakdown in associativity in those boundary gauge transformations to the group cohomology defining the bulk action. Recall from [70] [section 6.5] that the action associated to a 3-simplex such as that in figure 1 is given by a group cohomology 3-cocycle $\alpha(g, h, k)$. In fact, by imagining that the 3 -simplex contains a boundary just as in [73], we can identify the boundary phase with $\alpha(g, h, k)$.

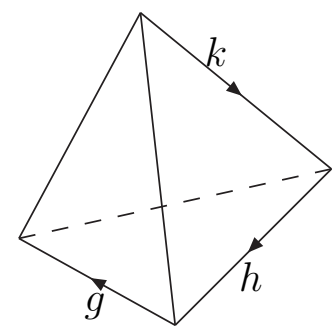

Figure 1: A 3-simplex.

The total action in the case that spacetime is a three-torus, for example, can be computed from a simplicial decomposition of $T^{3}$, and for fixed group elements on the boundaries is given in [70] [equ'n (6.35)] as

$$
\frac{\alpha(g, h, k) \alpha(h, k, g) \alpha(k, g, h)}{\alpha(g, k, h) \alpha(h, g, k) \alpha(k, h, g)} .
$$

This is the same phase factor that was computed for formal $C$-form analogues of discrete torsion in 75$]$. It is invariant under group coboundaries, as well as $S L(3, \mathbb{Z})$. The interpretation of the 3-cocycle $\alpha(g, h, k)$ can be read off from 12 [75]: it is precisely the obstruction to associativity in the $\mathbf{B} U(1)_{\text {conn }}$ transformations. Finally, note that although the nonlinear sigma model $M$ played a role in initially setting up our discussion, it is irrelevant to the result.

Putting this together, we see that in Dijkgraaf-Witten theory on a manifold with boundary, the gauge group $G$ can not act honestly on the boundary theory, but rather associativity of its group law is obstructed by an element of group cohomology $H^{3}(G, U(1))$, the same element defining the Dijkgraaf-Witten action.

\footnotetext{
${ }^{12}$ Although 75 was written to describe group actions on $C$ fields, with only minor modifications it applies to the present case, essentially by identifying the $B$ fields for various $g$ as the image of the boundaries, rather than as gauge transformations themselves.
} 
Now, although $G$ itself can not act honestly on the boundary theory, the element of $H^{3}(G, U(1))$ defines a 2-group extension

$$
1 \longrightarrow \mathrm{B} U(1) \longrightarrow \tilde{G} \longrightarrow G \longrightarrow 1
$$

which does act on the boundary theory - the obstruction to associativity indicates that one should replace $G$ by $\tilde{G}$ above.

It is worth mentioning that a closely related argument for ordinary discrete torsion in two dimensions appears in [73]. There, the fact that the group 2-cocycle defines a boundary phase factor was applied to derive an expression for a 2 -simplex

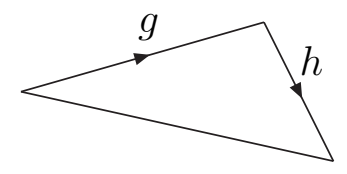

which was then applied to compute phase factors for Riemann surfaces of various genera, obtaining results matching standard discrete torsion phase factors. The conclusion there was analogous: the same element of $H^{2}(G, U(1))$ that defines discrete torsion, also defines an extension of $G$ that acts honestly on the boundary theory. In that case, the extension is an ordinary group extension, whereas here the extension that acts on the boundary theory, is a 2-group extension.

The same argument appears to imply that boundaries of higher-dimensional analogues of Dijkgraaf-Witten theory, defined by elements of $H^{d}(G, U(1))$ in $d$ bulk dimensions, should be acted upon by $(d-1)$-groups given as extensions

$$
1 \longrightarrow \mathbf{B}^{d-2} U(1) \longrightarrow \tilde{G} \longrightarrow G \longrightarrow 1
$$

Indeed, precisely this structure has been discussed elsewhere, see for example [41] [section $3.9 .130]$.

A different approach to Dijkgraaf-Witten theory and 2-groups is discussed in [11]. The approach there seems to be to extend Dijkgraaf-Witten theory to 2-groups, rather than consider boundaries in Dijkgraaf-Witten theory, as we have reviewed here. A related recent paper [76] describes $B^{p} G$ gauge theories, for $G$ finite.

\subsection{WZW models}

As seems to be very well-known in certain circles (see e.g. [8,41]), a different example of a theory with a type of one-form (2-group) action is provided by WZW models [77]. For 
applications later in this paper, we shall give a physics-oriented review of the highlights here. Recall that a WZW model in two dimensions is essentially a sigma model on a group manifold $G$ with a $B$ field with nonzero curvature, where the $B$ field is encoded in the Wess-Zumino term via its curvature as

$$
\int_{\Sigma} g^{*} B=\int_{Y} \operatorname{Tr}\left(g^{-1} d g\right)^{3}
$$

for a three-manifold $Y$ bounding $\Sigma$. (Strictly speaking, the Wess-Zumino term is defined up to addition of a closed 2 -form; locally,

$$
\int_{\Sigma} g^{*} B \sim \int_{\Sigma} g^{*}(B+d \Lambda)
$$

for some one-form $\Lambda$.) We claim that a WZW model on $G$ admits an action of the 2-group 14

$$
1 \longrightarrow \mathrm{B} U(1) \longrightarrow \tilde{G}_{1} \longrightarrow G \longrightarrow 1
$$

where the choice of extension of $G$ by $\mathbf{B} U(1)$ is the level of the WZW model. In this case, the stack underlying the 2 -group is the $U(1)$ gerbe whose connection $B$ is defined above.

As a quick consistency check, note that such extensions $\tilde{G}_{1}$ are classified by (appendix $\mathrm{A}$ ) elements of $H^{3}(G)$, which also classify the allowed levels of the WZW model (for simple simply-connected $G$, which we assume).

This 2-group is merely acting by automorphisms of the underlying gerbe. In this case, the 2-group is not merely " $G$-valued one-forms," but rather is more complicated.

Physically 15 , we can describe the action of $\tilde{G}_{1}$ by right- or left- multiplication on the fields of the WZW model as follows. First, describe a multiplication on $\tilde{G}_{1}$, i.e. a map $\tilde{G}_{1} \times \tilde{G}_{1} \rightarrow \tilde{G}_{1}$, by a pair $(h, A)$ consisting of a group element $h \in G$ and a connection $A$ on a principal $U(1)$ bundle over $G$. (The effect of multiplication by an element of $\tilde{G}_{1}$ is to induce an automorphism of $\tilde{G}_{1}$, and such automorphisms are essentially specified by pairs of the given form.) To describe the action on the WZW model, in closed string path integral quantization, we must specify the action on the classical map $g: \Sigma \rightarrow G$ ( $\Sigma$ the worldsheet) and on the WZW model $B$ field. These actions are as follows:

- $g \mapsto h g$ or $g h$ (depending upon whether a left- or right-multiplication was specified),

\footnotetext{
${ }^{13}$ In a nonlinear sigma model on a space $X$, a closed $B$ field defines an analogue of a $\theta$ angle, twisting nonperturbative sectors by phases given by the holonomy of the $B$ field on various 2-cycles, which is trivial only when $B$ is exact. Here, however, we assume $G$ is simple and simply-connected, in which case both $H^{2}(G, \mathbb{Z})$ and $H_{2}(G, \mathbb{Z})$ vanish.

${ }^{14}$ Because we want to preserve the $B$ field defined intrinsically by the Wess-Zumino term, we only consider 2-groups defined by extensions by $\mathbf{B} U(1)$, and not $\mathbf{B} U(1)_{\text {conn }}$.

15 Mathematically, the 2-group action on the gerbe is described in [29] [section 7.3], and its multiplication in terms of Wilson lines (i.e. on the loop space) was described explicitly in section 2.
} 


\section{- $B \mapsto B+F$, where $F$ is the curvature of $A$.}

The action on $g$ itself is identical to the $G$ action in an ordinary WZW model, and has been extensively discussed elsewhere. The action on $B$ leaves the curvature of $B$ invariant, and hence the Wess-Zumino term is unaffected. In principle, the $B$ field periods have changed by $\int g^{*} F$, but since $F$ lies in integral cohomology, and the $B$ field curvature is multiplied by an integer (the level), the closed string theory is invariant.

Put more simply, the action of $\tilde{G}_{1}$ on the fields of the WZW model is nearly identical to the action of $G$ itself, the difference being that the former includes an additional $B$ field transformation. The reason such a similar structure is possible is mathematically that the gerbe admits a canonical $G$-equivariant structure, and so $G$ has a natural action on the gerbe defining the WZW model. That said, the $B$ field transformations induce subtle differences in phases which manifest elsewhere. For example, if we apply the same reasoning to states defined on hemispheres, then the $B$ field transformations generate phase rotations on states, which the $G$ action by itself would not provide. Thus, in principle states in canonical quantization detect the difference between the actions of $G$ and $\tilde{G}_{1}$, via differences in phases. (Also, the $B$ field actions of the $\tilde{G}_{1}$ multiplication also manifest in D-brane actionas, as has been discussed in [78].)

Now, left- and right-multiplication by elements of $G$ is a global symmetry (though not a local symmetry) of the classical theory. However, in the WZW model, the algebra of their left- and right-moving Noether currents $J^{a}$ has a central extension, which in a dual theory of fermions would correspond to a two-point anomaly. As is well-known, the resulting symmetry algebra is a Kac-Moody algebra, of the form

$$
\left[J_{n}^{a}, J_{m}^{b}\right]=f^{a b c} J_{n+m}^{c}+\frac{1}{2} k n \delta^{a b} \delta_{n+m, 0},
$$

where the $f^{a b c}$ are the structure constants of the (finite-dimensional) Lie algebra. As a result of the central extension, the currents $J^{a}$ do not transform in a representation of the group $G$, unlike primary fields, but rather transform under the group action as [79][equ'n (3.17)]

$$
\delta_{\omega} J(z)=[\omega(z), J(z)]+\frac{1}{2} k \omega^{\prime}(z)
$$

for $\omega$ a Lie-algebra-valued function.

Those Kac-Moody algebras have a natural interpretation in terms of the 2 -group $\tilde{G}_{1}$, as infinitesimal automorphisms, as has been discussed in e.g. [8]. Now, Kac-Moody algebras are perhaps more widely understood in terms of the algebra of an extension of the algebra of the loop group $L G$ of $G$, but these are closely related. Specifically, the looping of the 2-group $\tilde{G}_{1}$,

$$
1 \longrightarrow \mathbf{B} U(1) \longrightarrow \tilde{G}_{1} \longrightarrow G \longrightarrow 1
$$


is precisely $\sqrt{16}$ the analogous central extension $\widetilde{L G}$ of the loop group $L G$,

$$
1 \longrightarrow U(1) \longrightarrow \widetilde{L G} \longrightarrow L G \longrightarrow 1 \text {. }
$$

(This is a special case of the statement that a $U(1)$ 1-gerbe over a space $X$ lifts to a principal $U(1)$ bundle over the loop space $L X$.) In this fashion, we can understand the more historically common interpretation of the Kac-Moody algebra, in terms of extensions of $L G$, as a looping of the 2-group $\tilde{G}_{1}$. Phrased differently, the de-looping of $\widetilde{L G}$, though not a group, is the 2-group $\tilde{G}_{1}$.

So far we have briefly reviewed how the 2 -group $\tilde{G}_{1}$ has a natural action on the WZW model by combining the $G$ action with $B$ field gauge transformations (in fact, the underlying $U(1)$ gerbe is the one that defines the Wess-Zumino term), and that the Kac-Moody algebra is the infinitesimal algebra of that same 2-group.

The reader might ask at this point about the Noether current associated with this 2-group $\tilde{G}_{1}$ and its left- and right-multiplications. Since the action of $\tilde{G}_{1}$ on the fields of the WZW model is, in the closed string theory, effectively indistinguishable from the action of $G$, the Noether currents for $\tilde{G}_{1}$ should be the same as that for $G$-namely, the Kac-Moody currents above. Put another way, we propose that the Kac-Moody currents have two interpretations: one interpretation as the Noether current of $G$ (with a central extension in the algebra), and another interpretation as the Noether current of $\tilde{G}_{1}$ (in which we propose that the central term be interpreted in terms of the $B$ field transformations in $\tilde{G}_{1}$, reflecting the phases picked up by states in canonical quantization).

As evidence for the interpretation above, we observe that a Noether current for the $B$ field transformations should be a two-form current, which would dualize in two dimensions to a scalar. We propose therefore that the central extension term in the Kac-Moody algebra is the Hodge dual of that two-form current, and that the entire Kac-Moody algebra be understood as the Noether current for $\tilde{G}_{1}$ multiplication. (The fact that the central extension is dually described in terms of an anomaly, will be interpreted later in this paper as an example of anomalies transmuting a classical group symmetry into a higher group symmetry of the quantum theory.)

To close an intellectual loop, it is natural to conjecture that, for suitable definitions, representations of the 2-group $\tilde{G}_{1}$ should coincide with representations of the corresponding KacMoody algebra, and indeed this conjecture has been made by others (see e.g. [80] [appendix A], [27]). After all, the states of a WZW model form representations of a Kac-Moody algebra, and we have also argued that the 2-group $\tilde{G}$ defines a global symmetry of the theory. Unfortunately, we are under the impression that the representation theory of 2-groups is not sufficiently well-developed to address this issue. (For current work on representations of

\footnotetext{
${ }^{16}$ See [8,22,23 for further discussion of the relationship between loop groups and 2-groups. It should be noted that for this discussion, we specifically need $\tilde{G}_{1}$ and not $\tilde{G}_{c}$, as the latter is not so closely related to the loop group extension.
} 
2-groups, see for example [25, 81, 82].)

In this language, it is tempting to speculate that level-rank duality should be realized as some sort of equivalence, perhaps a Morita equivalence [83], of 2-groups, for example as

$$
\widetilde{S U(n)_{k}}=\widetilde{S U(k)_{n}},
$$

where subscripts indicate levels.

Let us conclude this section by observing certain formal similarities between the structure in this section and our discussion of Dijkgraaf-Witten boundaries in section 3.3. There, given a bulk Dijkgraaf-Witten theory in three dimensions, corresponding to a finite $G$ gauge theory, we argued that any boundary theory should have a $\tilde{G}$ symmetry, where

$$
1 \longrightarrow \mathrm{B} U(1) \longrightarrow \tilde{G} \longrightarrow G \longrightarrow 1
$$

is the extension determined by the same element of $H^{3}(G, U(1))$ that determined the bulk theory. In this section, we have studied WZW models. However, WZW models can be understood as boundaries of three-dimensional Chern-Simons theories (see e.g. [84]). The Chern-Simons theory is a $G$ gauge theory, classified by an element of $H^{3}(G, \mathbb{Z})$ (the level), and we have argued that the boundary WZW theory admits a $\tilde{G}$ symmetry, where

$$
1 \longrightarrow \mathrm{B} U(1) \longrightarrow \tilde{G} \longrightarrow G \longrightarrow 1
$$

is the extension determined by the same element of $H^{3}(G, \mathbb{Z})$ that determined the bulk theory.

In the case of discrete torsion in two dimensions and Dijkgraaf-Witten theory in three dimensions, it was essential that the boundary theory couple to the bulk theory in such a way that the boundary form potential transform when the bulk gauge field undergoes a gauge transformation. Thus, the parallel would be especially meaningful if WZW models and Chern-Simons theories coupled in an analogous fashion. The reference [84 restricts to bulk gauge transformations that are trivial along the boundary, but if we consider more general cases, it is straightforward to see that under a gauge transformation, the ChernSimons action picks up an integral of a total derivative. In principle one could imagine that this could be cancelled by a symmetry transformation of the WZW $B$ field.

In passing, we should mention that such bulk/boundary relationships have been studied more generally in e.g. [2, 85, 86] in extended TQFT's, and also in related work [87, 89]. Roughly speaking, the form of the results is that a $\mathbf{B}^{k} G$ gauge theory in $d$ dimensions has on its boundary a theory with symmetry $\tilde{G}$ given as the extension

$$
1 \longrightarrow \mathbf{B}^{d-2} U(1) \longrightarrow \tilde{G} \longrightarrow \mathbf{B}^{k} G \longrightarrow 1 \text {. }
$$

As such examples have been discussed extensively elsewhere, and will play no further role in this paper, we will not discuss them further here. 


\subsection{Current algebras}

We have discussed how Kac-Moody currents in two-dimensional WZW models can be understood as Noether currents for a symmetry 2-group that mixes an ordinary group with $\mathrm{B} U(1)$.

In this section we shall conjecture an analogous role for current algebras in four dimensions.

In general, for two currents $J_{\mu, \alpha}, J_{\mu, \beta}$ ( $\mu$ a spacetime Lorentz index), their equal-time commutation relations will have the form [90] [section 22.6], [91][section 11-3]

$$
\left[J_{0, \alpha}(\vec{x}, t), J_{i, \beta}(\vec{y}, t)\right]=C_{\alpha \beta \gamma} J_{i, \gamma}(\vec{x}, t) \delta^{d-1}(\vec{x}-\vec{y})+S_{\alpha \beta, i, j} \partial_{j} \delta^{d-1}(\vec{x}-\vec{y}),
$$

where the second term on the right-hand-side is a c-number term known as the Schwinger term, and we have formally written the expression for general dimensions $d$, instead of specializing to $d=4$.

The Schwinger terms above have long been interpreted as defining a projectivization of the algebra of maps from the spacetime into a classical Lie algebra $\mathfrak{g}$ [92 95], and we propose a refinement of this idea. Specifically, we propose that the algebraic structure above be understood as a higher group extension of the form

$$
1 \longrightarrow \mathbf{B}^{d-1} U(1) \longrightarrow \tilde{G} \longrightarrow G \longrightarrow 1
$$

generalizing the structure of Kac-Moody algebras. (For example, if the four-dimensional spacetime is $\mathbb{R} \times T^{3}$, then a projectivization of maps into $\mathfrak{g}$ is the Lie algebra of a bundle on the triple loop group $L^{3} G$, and the triple loop space of the higher group above is exactly a bundle on $L^{3} G$.) The current for the $\mathbf{B}^{d-1} U(1)$ piece is a $d$-form, which dualizes in $d$ dimensions to a scalar. We conjecture that scalar should be interpreted as the Schwinger term. As a consistency check, note that extensions of the form above are classified by homotopy classes of maps 17

$$
\left[G, B B^{d-1} U(1)\right]=\left[G, B^{d} U(1)\right]=[G, K(\mathbb{Z}, d+1)]=H^{d+1}(G, \mathbb{Z})
$$

as discussed in appendix A. Indeed, in higher-dimensional analogues of WZW models as discussed in 18 e.g. [97], the analogue of the Wess-Zumino term in $d$ dimensions is defined by a degree $(d+1)$ form, integrated over a bounding $(d+1)$-dimensional space, which is precisely consistent with the classification above.

\footnotetext{
${ }^{17}$ For readers unfamiliar with the notation, $[X, Y]$ for $X, Y$ spaces or stacks denotes the homotopy classes of maps from $X$ to $Y$.

18 See also 96] for an updated description relevant for more general 4-manifolds. The analysis there interprets these structures in terms of differential cohomology theories. A thorough description of current algebras along the lines we suggest should take that into account, but as we are only outlining a conjecture, we shall not try to relate those differential cohomologies to higher groups in this brief section. Instead, we leave such an analysis for future work.
} 


\subsection{Other Kac-Moody actions}

We have seen that Kac-Moody algebras are closely related to Lie algebras of 2-groups, and so it is worth pointing out that there are other actions of Kac-Moody algebras in field theories in dimensions greater than two. We list a few examples below.

The work [98 101] described Kac-Moody actions on instanton moduli spaces (see also [102]), albeit at level 0. More recently, other Kac-Moody actions on four-dimensional gauge theories have been discussed in e.g. [103, 104, though again at level 0. As discussed in [105] [section 4], actions of Kac-Moody algebras on cohomology of moduli spaces of instantons on ALE spaces were discussed in e.g. [106].

Examples of hyperbolic Kac-Moody algebra actions on supergravity theories are discussed in a number of references including e.g. [107-111].

\section{Cosmological defects and generalized moduli spaces}

\subsection{Generalities on defects and ordinary moduli spaces}

In four dimensional theories, there are a variety of cosmological defects that are typically classified by the homotopy of the moduli space of scalar vacuum expectation values. For example, if $\mathcal{M}$ denotes that moduli space, then [112] [section 3.2]:

- $\pi_{0}(\mathcal{M})$ counts components of $\mathcal{M}$, hence counts possible domain walls,

- $\pi_{1}(\mathcal{M})$ counts possible cosmic strings, determined by winding in the scalar vevs,

- $\pi_{2}(\mathcal{M})$ counts analogues of monopoles, here determined by noncontractible two-spheres in $\mathcal{M}$, and

- $\pi_{3}(\mathcal{M})$ counts textures.

Examples often arise in gauge theories. Given a $G$ gauge theory with a Higgs field that breaks $G$ to $H \subset G$, and a suitable nontrivial potentia 19 , the moduli space $\mathcal{M}$ of Higgs vevs can be taken to be the coset $G / H$. The homotopy groups of $\mathcal{M}=G / H$ can be related to the homotopy groups of $G$ and $H$ using the homotopy long exact sequence, for example

$$
\pi_{k}(G) \longrightarrow \pi_{k}(G / H) \longrightarrow \pi_{k-1}(H) \longrightarrow \pi_{k-1}(G)
$$

\footnotetext{
${ }^{19}$ We are interested in topological defects, not semilocal strings. In the latter, the potential could vanish identically, so the full moduli space of Higgs vevs also includes e.g. vanishing Higgs vevs, or other vevs for which the unbroken subgroup is different from that above.
} 
We shall see applications of these ideas momentarily.

Now, let us outline a classification of analogues of topological defects pertinent to oneform symmetries and corresponding two-groups. We claim that the relevant analogue of the moduli space $20 \mathcal{M}$ of scalar field vevs is the loop space $L \mathcal{M}$ parametrizing Wilson lines. After all, Wilson line vevs are sections of a bundle over $L \mathcal{M}$, a bundle determined by the $\mathbf{B} G$ action, in the same way that for ordinary symmetries, the scalars are sections of a bundle over $\mathcal{M}$. More explicitly, (low-energy) Wilson lines depend upon scalars explicitly as, for example,

$$
P \exp \left(\int \phi^{a} A_{\mu}^{a} d s^{\mu}\right)
$$

hence a given Wilson line is determined by a loop in $\mathcal{M}$, in line with observations above about cosmic strings, and becomes a (not necessarily neutral) scalar over the (based) loop space $L \mathcal{M}$.

Then, just as homotopy of the space of scalar vevs $\mathcal{M}$ can encode information about topological defects pertinent to an ordinary group $G$, homotopy of the loop space $L \mathcal{M}$ of scalar field vevs can encode information about topological defects pertinent to $\mathrm{B} G$. To that end, there is a key identity relating the homotopy of $\mathcal{M}$ and its based loop space $L \mathcal{M}$ :

$$
\pi_{k}(\mathcal{M}) \cong \pi_{k-1}(L \mathcal{M})
$$

Alternatively, the reader might prefer to think about analogues of Wilson lines for $B G$ symmetries as defined by Wilson surfaces of the form

$$
\exp \left(\int_{\Sigma} F\right)
$$

for $\Sigma$ some two-dimensional submanifold and $F$ a two-form. Broadly speaking, these would be associated with elements of $\pi_{2}(\mathcal{M})$, but using the identity above,

$$
\pi_{2}(\mathcal{M}) \cong \pi_{1}(L \mathcal{M})
$$

and so again we can reduce these considerations to properties of the loop space.

Let us walk through the implications of this statement for Wilson lines for one-form symmetries:

\footnotetext{
${ }^{20}$ Or stack. See [56] for a discussion of cases in which the moduli 'space' admits a stack or gerbe structure, and in particular the relevance to cosmic strings and so forth of homotopy groups of the resulting gerbe. We should add that, although that reference did not make a clear statement regarding existence of cosmic strings and so forth associated with trivially-acting finite groups, we have since come to believe that they do exist, hence we now believe that homotopy groups of Deligne-Mumford stacks should indeed have physical relevance.
} 
- Analogues of domain walls for Wilson lines should in principle be counted by $\pi_{0}(L \mathcal{M})=$ $\pi_{1}(\mathcal{M})$, which correspond to cosmic strings. Here the intuition is simply that, as the cosmic string corresponds to a 't Hooft loop dual to the Wilson line [113, then the Wilson line changes as it winds around the cosmic string. Thus, in this sense, cosmic strings can define analogues of domain walls for Wilson lines.

For a simple example, consider the case of a $G$ gauge theory broken to $H \subset G$ by a Higgs vev, as outlined above. The cosmic strings are classified by $\pi_{1}(G / H)$. Now, if we run a Wilson line

$$
P \exp \left(\int_{C} A \cdot d s\right)
$$

around a cosmic string, by taking the curve $C$ to enclose the cosmic string, then we expect the phase of the Wilson line to be determined in part by the cosmic string. In other words, the gauge field along the Wilson line should couple to a bundle on $C$ induced by the cosmic string (as a 't Hooft line). Now, it is not a coincidence that, at least for $G$ connected and simply-connected, the classification of cosmic strings in this theory matches the classification of induced bundles on circles. Specifically, from the homotopy long exact sequence, if $G$ is connected and simply-connected, $\pi_{1}(G / H)=$ $\pi_{0}(H)$. Now, principal $H$ bundles on $S^{1}$ are classified by

$$
\left[S^{1}, B H\right]=\pi_{1}(B H)=\pi_{0}(H)
$$

so we see that the classification of cosmic strings matches that of principal $H$ bundles on a circle, as one would expect from the fact that the cosmic string (as a 't Hooft loop) is inducing a bundle on the enclosing circle. In particular, a cosmic string acts as a domain wall in the space of Wilson line vevs: depending upon whether the curve $C$ encloses a cosmic string (and the number of times it wraps), one should get a different component of the space of Wilson line vevs.

- Analogues of cosmic strings for Wilson lines should in principle be counted by $\pi_{1}(L \mathcal{M})=$ $\pi_{2}(\mathcal{M})$, which correspond to monopoles. To help explain why this is sensible, first recall that a cosmic string can be interpreted as a 't Hooft line [113]. A charged particle that walks along a loop enclosing the 't Hooft line will pass through a transition function for a nontrivial bundle generated by the 't Hooft line. The analogue of a 't Hooft line for a $B G$ symmetry is a monopole, which generates a nontrivial $G$-gerbe. Indeed, as one drops a monopole through a Wilson line, its vev will change21, so in this fashion one has a notion of winding for Wilson lines. A Wilson line walking through a loop in which it wraps a monopole is schematically illustrated in figure 2.

As a particular example, let us consider analogues of Alice strings. Recall (see e.g. [112] [section 4.2.4], [113 116]), Alice strings arise in $S O(3)$ (and $S U(2)$ ) gauge theories

\footnotetext{
${ }^{21}$ See for example [29] [section 7.1], but in fact at some level this is merely an unwinding of the definition of a monopole across the surface of an enclosing $S^{2}$.
} 


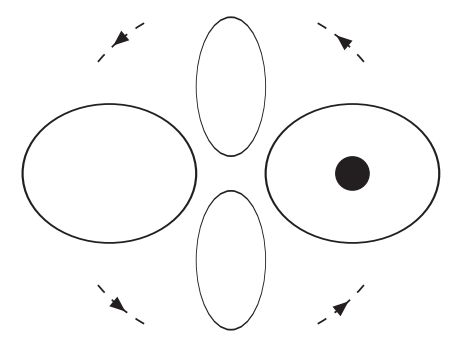

Figure 2: A Wilson line about a closed loop itself moves through a closed path, which encloses a monopole (right side).

with matter in the 5 -dimensional representation, consisting of real symmetric traceless $3 \times 3$ matrices. A typical Higgs vev, e.g.

$$
\left[\begin{array}{ccc}
1 & 0 & 0 \\
0 & 1 & 0 \\
0 & 0 & -2
\end{array}\right]
$$

has stabilizer $S(O(2) \times O(1))=O(2) \subset S O(3)$, so with a suitable potential forcing the vacua to lie on the coset, the moduli space of Higgs vevs is $S O(3) / O(2)=\mathbb{R P}^{2}$. (Equivalently, we could work in an $S U(2)$ gauge theory, in which case the stabilizer of the Higgs vev above would be ${ }^{22} \operatorname{Pin}(2) \subset S U(2)$, and again $S U(2) / \operatorname{Pin}(2)=\mathbb{R P}^{2}$.) This moduli space has $\pi_{1}=\mathbb{Z}_{2}$, hence there are cosmic strings in this model, in which the Higgs field vev wraps nontrivially around the moduli space. More to the point, the cosmic strings are classified by $\mathbb{Z}_{2}$. The Wilson lines act on electric charges of the unbroken $O(2)(\operatorname{Pin}(2)$ in the $S U(2)$ theory) by flipping their signs: representations of $O(2)$ (resp. Pin(2)) are pairs of matched $S O(2)$ (resp. Spin(2)) representations of opposite sign [117] [section 11.1], and the Wilson line about the string, which takes values in the diconnected component, flips the signs, exchanging the two $S O(2)$ (resp. Spin(2)) representations. The fact that electric charges undergo a sign flip under parallel transport about the string, ultimately a consequence of the fact that the strings are classified by $\mathbb{Z}_{2}$, is the defining characteristic 23 of Alice strings.

The analogue of Alice strings for Wilson lines would involve a moduli space $\mathcal{M}$ such

\footnotetext{
${ }^{22} \operatorname{Pin}(2)$ here can be understood as the normalizer of a maximal torus. Pin(2) is also a double-cover of $O(2)$.

${ }^{23}$ As an aside for interested readers, let us discuss another characteristic feature of Alice strings in this language. Take a $S O(2)$ charge $q$, and pass through the middle of a pair of parallel Alice strings. Asymptotically, the pair of parallel Alice strings yield an $O(2)$ bundle whose disconnected component is trivializable, so $S O(2)$ charges are sensible asymptotically (though not invariant under $O(2)$ gauge transformations). Pass a charge $q$ through the middle of the pair, and it will come out on the other side as charge $-q$, hence to preserve charges, the pair of Alice strings are then interpreted as having charge $+2 q$. This phenomenon is known as 'Cheshire charge.' The basic issue in all these cases seems to be one of ill-fated attempts to define $S O(2)$ matter in an $O(2)$ gauge theory.
} 
that $\pi_{1}(L \mathcal{M})=\pi_{2}(\mathcal{M})=\mathbb{Z}_{2}$. In general, for a $G$ gauge theory with a potential forcing all the Higgs vevs to break $G$ to the same subgroup $H \subset G$, the moduli space of Higgs vevs is the coset $G / H$. For example24, consider an $S U(3)$ gauge theory in which a Higgs vev breaks the $S U(3)$ to $S O(3) \subset S U(3)$. This would happen for a Higgs field in the $\operatorname{Sym}^{2} \mathbf{3}=\mathbf{6}$ representation, with a vev given by the identity. This theory has the property that

$$
\pi_{2}(S U(3) / S O(3))=\mathbb{Z}_{2} .
$$

In particular, a Wilson line following a path that swoops around a monopole, as in figure 2, would undergo a gauge transformation in the nontrivial element of $\mathbb{Z}_{2}$.

- Analogues of monopoles for Wilson lines should in principle be counted by $\pi_{2}(L \mathcal{M})=$ $\pi_{3}(\mathcal{M})$, which correspond to textures.

- Finally, analogues of textures for Wilson lines should in principle be counted by $\pi_{3}(L \mathcal{M})=\pi_{4}(\mathcal{M})$.

Another standard matter involves defects ending on other defects. For example, suppose a gauge symmetry $G$ is broken in two steps at successively lower energies, first to a subgroup $H \subset G$, and then $H$ is completely broken. (See e.g. 411][section 5.7.1] for a longer review.)

- Domain walls ending on strings: After the first step, when $G$ is broken to $H$, the theory will have cosmic strings counted by $\pi_{1}(G / H)$. After the second step, the theory will have domain walls counted by $\pi_{0}(H)$. The domain walls of the second step can end on the strings of the first step, consistent with the observation that (for $G$ connected and simply-connected), $\pi_{1}(G / H) \cong \pi_{0}(H)$.

- Strings ending on monopoles: After the first step, when $G$ is broken to $H$, the theory will have monopoles counted by $\pi_{2}(G / H)$. After the second step, the theory will have strings counted by $\pi_{1}(H)$. The strings of the second step can end on the monopoles of the first step, consistent with the observation that (for $G 1$ - and 2connected $), \pi_{2}(G / H) \cong \pi_{1}(H)$.

The analogue for Wilson lines is similar:

- Domain walls ending on strings: After the first step, when $G$ is broken to $H$, the theory will have cosmic strings counted by $\pi_{1}(L G / H)=\pi_{2}(G / H)$. After the second step, the theory will have domain walls counted by $\pi_{0}(L H)=\pi_{1}(H)$. Thus, the analogue of domain walls ending on strings, can equivalently be understood as ordinary strings ending on ordinary monopoles.

\footnotetext{
${ }^{24}$ We would like to thank A. Knutson for suggesting this example.
} 
In principle, the same analysis can be continued for higher $p$-form symmetries. For example, analogues of cosmological defects for a Wilson surface corresponding to a 2-form symmetry would be measured by $\pi_{k}\left(L^{2} \mathcal{M}\right)=\pi_{k+2}(\mathcal{M})$. For example, a domain wall for a Wilson surface would correspond to an ordinary monopole: the set of values for a Wilson surface would break into components, determined by how many times the Wilson surface wraps the monopole.

In a similar fashion, analogues of cosmological defects for a Wilson surface corresponding to a $p$-form symmetry would be measured by $\pi_{k}\left(L^{p} \mathcal{M}\right)=\pi_{k+p}(\mathcal{M})$. Of course, for a spacetime of a given dimension, there is an upper limit to the degree of homotopy groups that can be realized in that spacetime. The intuition behind higher examples rapidly becomes obscure, so we will not pursue this further, except to note that this does provide a physical interpretation to higher homotopy groups of the moduli space $\mathcal{M}$ of scalar field vevs.

\subsection{Generalized moduli spaces}

So far we have discussed cosmological defects and higher loops for ordinary moduli spaces. Next, we shall consider cases in which a higher group action exists on the moduli space of the field or string theory. In examples in which the moduli 'space' admits an action of a higher group, the 'space' is a generalized space known as a stack.

The paper [56] studied a number of properties of four-dimensional theories whose moduli 'spaces' were of this form. For completeness, we begin by reviewing the highlights of cosmological defects studied in [56], here. We will then turn to other moduli 'spaces' appearing in field and string theory, motivated by our discussion of higher group actions on WZW models and current algebras.

To begin, consider as a prototypical example a four-dimensional $U(1)$ gauge theory with 2 chiral multiplets of charge $+k$ and 2 chiral multiplets of charge $-k$, for $k>1$, along with massive minimally-charged matter. As discussed earlier in section 3.2 as well as e.g. [51 53, 56, 57], both the quantum field theory and the moduli 'space' admit an action of $\mathbf{B} \mathbb{Z}_{k}$, and so its spectrum of cosmological defects is slightly different than for ordinary cases.

Let $\mathcal{M}$ denote the moduli 'space' of the theory above, and $M$ the ordinary moduli space of the corresponding theory with $k=1$. As discussed in [56], there is a long exact sequence of homotopy groups

$$
\cdots \longrightarrow \pi_{m}\left(\mathbf{B} \mathbb{Z}_{k}\right) \longrightarrow \pi_{m}(\mathcal{M}) \longrightarrow \pi_{m}(M) \longrightarrow \pi_{m-1}\left(\mathbf{B} \mathbb{Z}_{k}\right) \longrightarrow \cdots
$$

In principle, one would expect cosmological defects in the theory with $k>1$ to be counted by $\pi_{m}(\mathcal{M})$, rather than $\pi_{m}(M)$. In the case above, the theory with $k=1$ admits only

${ }^{25}$ This assumes that e.g. cosmic strings defined by nontrivial bundles for trivially-acting groups exist and 
monopoles, as only $\pi_{2}(M) \neq 0$. The corresponding theory with $k>1$ also admits monopoles, but the counting is slightly different. In particular, since $\pi_{2}\left(\mathbf{B} \mathbb{Z}_{k}\right)$ vanishes, the homotopy sequence above reduces to

$$
0 \longrightarrow \pi_{2}(\mathcal{M}) \longrightarrow \pi_{2}(M)(\cong \mathbb{Z}) \longrightarrow \pi_{1}\left(\mathbf{B} \mathbb{Z}_{k}\right)\left(\cong \mathbb{Z}_{k}\right) \longrightarrow 0 .
$$

Thus, our theory with $k>1$ also admits countably many monopoles, but counted slightly differently than in the case $k=1$. In particular, we see that not every monopole in the $k=1$ theory lifts to a monopole in the $k>1$ theory - apparently only monopoles whose charges satisfy a divisibility constraint lift to the $k>1$ theory, as one might expect on general principles of charge quantization.

As one more example, consider a four-dimensional theory in which one gauges a finite group $G$, all of which acts trivially on the theory. If the ungauged theory had moduli space $M$, then the gauge theory has moduli 'space' $M \times \mathbf{B} G$. In this case, the counting is again modified, but in a more trivial fashion, as

$$
\pi_{1}(M \times \mathbf{B} G)=\pi_{1}(M) \oplus \pi_{1}(\mathbf{B} G)
$$

and other homotopy groups are unmodified. Again the counting of cosmological defects is modified, but in a much more trivial fashion.

So far we have considered moduli 'spaces' of four-dimensional field and string theories admitting an action of $\mathbf{B} G$ for $G$ finite. Mathematically, there is a notion of generalized 'spaces' that would admit actions of $\mathbf{B} G$ for $G$ nonfinite; however, it is not known at present how to, for example, define a sigma model on such. (Technically, it is known how to define a sigma model on a Deligne-Mumford stack, but not an Artin stack.) In the remainder of this section, we will explore the possibility of whether such more general moduli 'spaces' might appear in physics via a simple example. We will get rather confusing results, suggesting that possibly the only generalized moduli 'spaces' relevant for physics are of the former (Deligne-Mumford) type.

In section 3.4 we reviewed existing results on how WZW models are believed to admit an action of $\tilde{G}$, an extension of a Lie group $G$ (partially defining the WZW model) by $\mathbf{B} U(1)$, where the extension class is determined by the level of the WZW model. We could imagine fibering the WZW model over some other space $X$, as described in e.g. [119 124]. The resulting two-dimensional theory has a semiclassical moduli 'space' given by a $\tilde{G}$-bundle over $X$, another example of a stacky moduli space, albeit an Artin stack and not a DeligneMumford stack.

are different from ordinary cosmic strings, in other words that $\pi_{1}\left(\mathbf{B} \mathbb{Z}_{k}\right)$ is counting cosmic strings of this type. At the time reference [56] was written, this matter was ambiguous, but now seems to have been settled in the affirmative. Such cosmic strings are defined by nontrivial $\mathbb{Z}_{k}$ bundles over enclosing circles, but in which the $\mathbb{Z}_{k}$ acts trivially on the other fields of the theory. (In particular, a map from any space $X$ into $\mathbf{B} \mathbb{Z}_{k}$ is defined by a $\mathbb{Z}_{k}$ bundle on $X$, both for the classifying space and for the stack, which is part of the reason why cosmic strings on such higher groups would have the form described.) 
The two-dimensional example above would, for obvious reasons, not admit a wide variety of cosmological defects, but formally we can imagine higher-dimensional analogues. Following the proposal in section 3.5 for an interpretation of current algebras in terms of higher groups, one could similarly imagine fibering a higher-dimensional current algebra over some space $X$, at least in low-energy effective field theory. If $\tilde{G}$ is the higher symmetry group of the current algebra, then by analogy with fibered two-dimensional cases, one would have a moduli 'space' given by a $\tilde{G}$-bundle over $X$.

In such a case, since $\tilde{G}$ was determined by an extension encoding an anomaly, in effect some of the homotopy groups of $\tilde{G}$ and hence of the moduli stack would be determined by an anomaly. Put another way, one would have anomaly-induced cosmological defects.

For example, let us consider the $S U(3)$ current algebra described in [97]. In present language, section 3.5 conjectures that it should have a $\tilde{G}$ symmetry, where $\tilde{G}$ is given by

$$
1 \longrightarrow \mathbf{B}^{3} U(1) \longrightarrow \tilde{G} \longrightarrow S U(3) \longrightarrow 1
$$

where the level $k$ of the current algebra ( $n$ in the notation of [97]) determines the extension class. We can consider this model by itself, in effect fibering over a point.

From the long exact homotopy sequence (which operates for higher groups in essentially the same way as for spaces [28]), we see that for $m \neq 4,5, \pi_{m}(\tilde{G})=\pi_{m}(S U(3)$ ), which (for $n \neq 4,5)$ is nonzero for $n=3$ and $n=7$ for example. For $m=4,5$, there is a long exact sequence

$$
0 \longrightarrow \pi_{5}(\tilde{G}) \longrightarrow \pi_{5}(S U(3))(\cong \mathbb{Z}) \longrightarrow \pi_{4}\left(\mathbf{B}^{3} U(1)\right)(\cong \mathbb{Z}) \longrightarrow \pi_{4}(\tilde{G}) \longrightarrow 0 .
$$

The map $\pi_{5}(S U(3)) \rightarrow \pi_{4}\left(\mathbf{B}^{3} U(1)\right)$ should be proportional to the level $k$ of the current algebra, so that at level zero, the sequence splits, and $\pi_{5}(\tilde{G}) \cong \pi_{5}(S U(3))$. If the level $k \neq 0$, then $\pi_{5}(\tilde{G})=0$ and $\pi_{4}(\tilde{G})=\mathbb{Z}_{k}$.

Unfortunately, it is not clear how to interpret the homotopy groups of the Artin stack above physically. It is possible that one must perform a more subtle analysis along the lines of [96]. However, for the moment we will instead interpret this to mean that the physical relevance of Artin stacks is unclear, and when computing e.g. cosmological defects, one should restrict to cases involving Deligne-Mumford stacks (meaning, actions of $\mathbf{B} G$ for $G$ finite).

\subsection{Analogues of Goldstone's theorem and generalized moduli spaces}

Previously in section 3.1 and in [1], analogues of Goldstone's theorem were discussed for counting Goldstone bosons in theories with spontaneously broken higher group symmetries. 
For example, the Goldstone boson associated with a spontaneously broken $\mathbf{B}_{b} U(1)_{\text {conn }}$ in an abelian gauge theory was proposed in [1] to be the gauge field itself.

Now, for ordinary groups, there is additional content in Goldstone's theorem. In principle, Goldstone's theorem not only gives a count of bosons associated with a spontaneously broken global symmetry, but also describes the local dimension of the moduli space. After all, if a global symmetry $G$ is broken to a subgroup $H$, then one has $\operatorname{dim} G / H$ massless bosons ultimately because the moduli space of vacua locally looks like $G / H$.

We have already discussed how generalized moduli 'spaces' (stacks) can admit actions of higher groups, so it is natural to ask what analogous statements can be made for the moduli stacks appearing in such gauge theories. For example, if one spontaneously breaks a $\mathbf{B} G$ symmetry to a subgroup $\mathbf{B} H$, then does a coset of the form $\mathbf{B} G / \mathbf{B} H$ play a role in physics, in the same way that for ordinary groups, cosets $G / H$ play a role in understanding moduli spaces in spontaneous symmetry breaking? In this section, we will not reach any firm conclusions, but we will discuss these issues in simple examples.

Let us first outline a little pertinent mathematics. If $\tilde{G}$ is a higher group, and $\tilde{H}$ a sub(higher)group, then the homotopy quotient $\tilde{G} / \tilde{H}$ exists [28], and there is a long exact sequence of homotopy groups of exactly the same form as for ordinary groups [28], namely

$$
\pi_{k}(\tilde{G}) \longrightarrow \pi_{k}(\tilde{G} / \tilde{H}) \longrightarrow \pi_{k-1}(\tilde{H}) \longrightarrow \pi_{k-1}(\tilde{G})
$$

For the higher groups $\mathbf{B}^{k} G$, we can use the fact that

$$
\pi_{m}\left(\mathbf{B}^{k} G\right)=\pi_{\pi_{m-1}}\left(\mathbf{B}^{k-1} G\right)=\cdots=\pi_{m-k}(G)
$$

to relate the homotopy groups of $\mathbf{B}^{k} G$ to those of $G$. The 'spaces' of such higher groups can be understood as stacks, or 'generalized spaces' in the language of the previous section.

Second, it may be pertinent to distinguish two cases. As previously outlined, stacks associated with $\mathbf{B} G$ for $G$ finite are different both mathematically and physically from those associated with $\mathbf{B} G$ for $G$ non-finite. For the former, known technically as Deligne-Mumford stacks, it is known how to define a sigma model [53. For the latter, known technically as Artin stacks, the physics is largely unknown, and we suspect likely to be more subtle than for Deligne-Mumford stacks. As a result, it is entirely possible that physical interpretations of homotopy groups of gerbe structures may differ between such cases.

Now, let us consider a concrete example. As was reviewed in section 3.1, reference [1] discusses abelian gauge theories as examples of theories with $\mathbf{B}_{b} U(1)_{\text {conn }}$ actions. As discussed there, in some dimensions, this symmetry is spontaneously broken, and the abelian gauge field is identified with the Goldstone boson. Let us try to interpret this result in terms of possible moduli stack structures.

- First, let us proceed naively, and look for an interpretation of the homotopy groups of $\mathbf{B}_{b} U(1)_{\text {conn }}$ itself. Now, as outlined in section 2, the homotopy groups of $\mathbf{B}_{b} U(1)_{\text {conn }}$ 
are the same as those of $K(U(1), 0)$, which is to say,

$$
\pi_{0}\left(\mathbf{B}_{b} U(1)_{\text {conn }}\right)=U(1), \pi_{n}\left(\mathbf{B}_{b} U(1)_{\text {conn }}\right)=0 \text { for } n>0 .
$$

However, it is not entirely clear to us how these homotopy groups would be interpreted in an abelian gauge theory.

- Next, let us take a slightly more radical approach. Since we have an abelian gauge theory, it is tempting to replace the moduli space of the theory $M$ with an Artin stack, a $U(1)$ gerbe over $M$ with fibers $\mathbf{B} U(1)$. This would be in the spirit, though not the detailed method, of [51, 53, 56]: a sigma model on a stack of this form should morally involve a path integral over $U(1)$ bundles with connection, hence an abelian gauge theory, though previous work has only made claims about stacks involving finite gerbes (Deligne-Mumford stacks). Sigma models on Artin stacks have not been studied at all, and are likely to be subtle to interpret. That said, if this extremely naive approach were to hold water, then in principle we could effectively encode the $U(1)$ gauge fields in the 'stacky' structure of the moduli space, consistent with the naive application of a generalized Goldstone's theorem as giving B $U(1)$ directions in the moduli 'space' in the spontaneously broken symmetry phase. That said, very naively, similar statements could also be made in other dimensions, even in which the large-loop behavior changes and one no longer has an IR interpretation in terms of spontaneous symmetry breaking.

At this point in time, we are therefore not able to make any firm statements.

\section{Anomalies as transmutation}

\subsection{Two-dimensional cases}

Let us now turn to the application of 2-groups to anomalies in two-dimensional quantum field theories. If $J$ is a Noether current, or rather the corresponding composite operator in some two-dimensional quantum field theory, then the $J^{2}$ anomaly in two dimensions is well-known to be proportional to $\operatorname{Tr} F \wedge F$, involving the curvature of a coupled (possibly nondynamical) gauge field. Anomalies of this sort are indicative of curvature on the space of gauge transformations, or somewhat more precisely, indicate that the path integral measure fails to be well-defined under families of gauge transformations. (See for example [125] [section 3] for a pertinent discussion.) This can happen even if there are no chiral fermi zero modes: the path integral measure can be invariant under a single gauge transformation, but not well-behaved in families.

The fact that the anomaly arises in families of gauge transformations suggests an interpretation of the anomaly. Although, the anomaly 'breaks' the classical symmetry group 
$G$, the form in which it is broken suggests that the quantum theory might have a different symmetry 'group,' perhaps one in which associativity is broken up to higher cocycles, where the associators are encoded implicitly in the phases one picks up around loops.

In this section, we will propose that in such cases, the quantum theory has a symmetry 2group, instead of a symmetry group, specifically the 2-group $\tilde{G}_{1}$ defined in [6] by an extension

$$
1 \longrightarrow \mathrm{B} U(1) \longrightarrow \tilde{G} \longrightarrow G \longrightarrow 1
$$

In particular, in this interpretation, the anomaly breaks the classical symmetry, but simultaneously encodes an extension of $G$ to a 2-group symmetry, and so describes a transmutation of a zero-form symmetry into a 2-group symmetry, a structure we have seen elsewhere in this paper.

One way to see this structure is via a dual representation of the fermions, in a WZW model. As is well-known [77], the nonabelian bosonization of fermions with classical symmetry group $G$ is described by a WZW model, and we described previously in section 3.4 how WZW models form examples of theories in which ordinary group actions are replaced with 2-group actions.

In this language, the fermion chiral symmetry currents correspond to, in the WZW model, classically the left and right multiplications by $G$. These classical symmetries are modified in the quantum theory by the central extension term in the Kac-Moody algebra, which, as has been argued here and elsewhere, suggests they should be extended to left and right multiplication by the 2 -group $\tilde{G}_{1}$, defined by the extension of $G$ by $\mathbf{B} U(1)$ dictated by the level of the WZW model. The fact that the 2-group $\tilde{G}_{1}$ explicitly appears in the bosonized representation of the fermions, argues that it must also appear in the quantized fermion theory, and since in the WZW model the 2-group $\tilde{G}_{1}$ extends the classical group $G$, in the dual fermion theory the 2 -group $\tilde{G}_{1}$ must replace the anomalous action of the classical symmetry group $G$.

Another check of this proposal arises when we couple the fermions to a gauge field. In principle, if the classical symmetry group becomes in the quantum theory a symmetry 2group, then to gauge $G$, any classical principal $G$ bundle $P$ must be lifted to a principal $\tilde{G}_{1}$ bundle. The obstruction to this lift 26 is discussed in [6]; it is of the form

$$
k \operatorname{Tr} F \wedge F
$$

where $F$ is the curvature of $P$ and $k \in H^{3}(G, \mathbf{Z})$ is the level of the affine algebra, or equivalently the cohomology class describing $\tilde{G}_{1}$ as an extension of $G$. (For example, for

\footnotetext{
${ }^{26}$ Obstructions to spin structures may be more familiar to some readers, and are analogous [118] [section 3]. Given a principal $S O(n)$ bundle $P$ over some manifold $M$, as is well-known there is an obstruction to lifting to a principal $\operatorname{Spin}(n)$ bundle, measured by the second Stiefel-Whitney class $w_{2}(P)$. Corresponding to this obstruction is a $\mathbb{Z}_{2}$ gerbe on $M$, whose characteristic class is given by $w_{2}(P) \in H^{2}\left(M, \mathbb{Z}_{2}\right)$.
} 
a trivial extension, $k=0$, and there is no obstruction.) Demanding that this obstruction vanish for all bundles $P$ is, in general, not possible, and so gauging the classical symmetry is not possible, as one expects for an anomalous symmetry.

Another check arises in fibered WZW models [119 124], describing e.g. a bosonization of the fermions in a nonlinear sigma model. The fibering of the WZW model is described by a principal $G$ bundle $P$ over the target space. Given a principal $G$ bundle $P$, there is an obstruction to lifting it to a principal $\tilde{G}_{1}$ bundle. For the same reasons as above, that obstruction is

$$
k \operatorname{Tr} F \wedge F,
$$

where $k \in H^{3}(G, \mathbf{Z})$ is the level of the WZW model, or equivalently the cohomology class describing $\tilde{G}_{1}$ as an extension of $G$. (For example, for a trivial extension, $k=0$, and there is no obstruction.) In a heterotic theory, this obstruction corresponds to a contribution to the Green-Schwarz anomaly in a $(0,2)$ theory describing a left-moving current algebra at level $k$, for example.

Consider more general fibered WZW models, in which one bosonizes both left and rightmovers. Suppose one has classically a principal $G_{L} \times G_{R}$ bundle, with different groups for left and right-movers. If we call this bundle $P$, then anomaly cancellation in this case becomes the statement that the $G_{L} \times G_{R}$ bundle can be lifted to a $\widetilde{G_{L} \times G_{R 1}}$ bundle; obstructions on either side separately are cancelled out when one takes both factors together.

The idea that a classical group can transmute into a 2-group symmetry of the quantum theory, as we have formulated it above, may seem odd, but analogous transmutations may also sometimes appear along renormalization group flows. For example, in two dimensions it is a common result that global symmetry groups become enhanced at IR fixed points to affine algebras, which as previously discussed are linked to 2-groups. In this language, at least naively RG flow would appear to describe a transmutation from a (UV) global symmetry group to an (IR) 2-group.

\subsection{Conjectures on elliptic genera}

We can gain further possible insight into the physical relevance of 2-groups by considering elliptic genera. The basic Witten genus describes a quantum theory of a set of fermions with a Spin rotation symmetry, fibered over some space $X$, coupled via the tangent bundle.

In discussions of elliptic genera, it is well-known that a topological group named String plays an important role. For this discussion, we should point out that $\operatorname{String}(n)$ can be built as a 2-group, extending $\operatorname{Spin}(n)$ at level one by $\mathbf{B} U(1)$ [6] [section 4.3], [7] [section 7], [8]:

$$
1 \longrightarrow \mathrm{B} U(1) \longrightarrow \operatorname{String}(n) \longrightarrow \operatorname{Spin}(n) \longrightarrow 1 \text {. }
$$


With the above in mind, it is very tempting to conjecture that the states appearing in elliptic genera form a representation of the 2-group $\operatorname{String}(n)$. In particular, the states lie in representations of an affine extension of $s o(n)$, and as we discussed in section 3.4, it is tempting to identify representations of the 2-group and the affine algebra. However, as we discussed there, the representation theory of 2-groups does not seem to be sufficiently welldeveloped at the moment to completely answer this question one way or another, so we leave this matter for future work..

\subsection{Higher-dimensional conjectures}

In $2 n$ dimensions, anomalies can arise from higher-dimensional analogues of the same issue with well-definedness in families. As discussed in e.g. [125] [section 3], consider a theory of charged fermions on $S^{2 n}$, coupling to a principal $G$ bundle $P$ over some space $X$. Under a one-parameter family of gauge transformations, the fermion path integral may fail to be well-defined, measured at least in part by an element of $H^{2 n+1}(G)$.

Proceeding solely by analogy with the two-dimensional case discussed above, in our language, this might correspond to the statement that the correct symmetry group of the theory is a $2 n$-group, which topologically is a $(2 n-1)$-gerbe over $G$, an extension of $G$ by $\mathbf{B}^{2 n-1} U(1)$. If we take enough loops, this becomes a central extension of $L^{2 n-1} G$ by $U(1)$. It is natural to conjecture that obstructions to lifting principal $G$ bundles to principal $\tilde{G}_{1}$ bundles, for $\tilde{G}_{1}$ a higher group extension as above, are characterized by a degree $2 n+2$ characteristic class, of the form

$$
k \operatorname{Tr} F \wedge \cdots \wedge F
$$

( $n+1$ factors), which would certainly tie into the description of anomalies, as well as central extensions in higher dimensional current algebras as outlined in section 3.5. We will leave this matter for future work.

\section{Conclusions}

In this paper we described the $p$-form symmetries of [1] as special cases of higher group actions in quantum field theory. After briefly outlining the definition of higher groups, we outlined several examples, both reviewing some already in the literature as well as outlining some new examples. We discussed the role of 'generalized moduli spaces' in quantum field theory, admitting actions of higher groups, and discussed cosmological defects in this context. We concluded with a proposal for an interpretation of certain anomalies in terms of higher group symmetries of quantum theories.

There is also evidence for a role for 2-groups in moonshine, see e.g. [126], [82] [section 
5]. It has also been suggested that such higher groups might play a further role in the understanding of Mathieu groups in SCFT's as described in e.g. [127 131], as for example the Mathieu group $M_{12}$ is a subgroup of a groupoid denoted $M_{13}$ [27,132].

In section 3.2, we discussed two-dimensional gauge theories in which a finite subgroup $G$ of the gauge group acts trivially on the massless matter. We argued that at low energies, these theories have a $B G$ symmetry, and outlined how these theories decompose into disjoint

unions of simpler theories. It would be interesting to understand if there are analogues of decomposition for any notion of $B G$ gauge theories or other higher gauge theories in any dimension.

It is tempting to speculate that some of the relations we have explored between ordinary groups and higher groups may have analogues outlined in [133], but we shall leave such considerations for future work.

\section{Acknowledgements}

We would like to thank M. Ando, N. Aramian, N. Epa, D. Freed, N. Ganter, A. Knutson, T. Pantev, U. Schreiber, and R. Thorngren for useful conversations. This paper began as an joint effort with M. Ando, who provided initial ideas and commentary, and N. Ganter and N. Epa similarly contributed to the development at an intermediate stage. U. Schreiber contributed many references and results on higher groups, and N. Aramian also contributed many technical observations. Although we were motivated to write up our results by the publication of [1, this paper is based on discussions of applications of 2-groups to physics that took place over several years, during which time E.S. was supported by NSF grants DMS-0705381, PHY-0755614, PHY-1068725, and PHY-1417410.

\section{A Topological classification of extensions}

In this paper we have encountered a number of higher groups $\tilde{G}$ constructed as extensions of the form

$$
\begin{gathered}
1 \longrightarrow \mathbf{B}^{k} U(1) \longrightarrow \tilde{G}_{1, k} \longrightarrow G \longrightarrow 1, \\
1 \longrightarrow \mathbf{B}^{k} U(1)_{\mathrm{conn}} \longrightarrow \tilde{G}_{c, k} \longrightarrow G \longrightarrow 1, \\
1 \longrightarrow B^{k} U(1) \longrightarrow \tilde{G}_{0, k} \longrightarrow G \longrightarrow 1,
\end{gathered}
$$

for some other (higher) group $G$. To classify the possible extensions, we first need to compute the topological classes of extensions. 
An extension of the form above is the total space of a $\mathbf{B}^{k} U(1)$ (or $\mathbf{B}^{k} U(1)_{\text {conn }}$, or $B^{k} U(1)$ ) bundle over $G$. Regardless of our version of $B^{k} U(1)$, for the purposes of a topological classification of bundles, we can replace it with a suitable Eilenberg-Maclane space:

$$
B^{k} U(1)=K(\mathbb{Z}, k+1), \quad B U(1) \cong \mathbb{C P}^{\infty} .
$$

(In particular, since the geometric realizations of all three options are homotopic to one another 27 , the resulting topological classification will not depend upon whether we are extending by $\mathbf{B}^{k} U(1)$ or $\mathbf{B}^{k} U(1)_{\text {conn }}$ or $B^{k} U(1)$.) Over any space $M, B^{k} U(1)=K(\mathbb{Z}, k+1)$ bundles are classified by homotopy classes of maps into a next higher Eilenberg-Maclane space, i.e. homotopy classes of maps

$$
M \longrightarrow K(\mathbb{Z}, k+2)
$$

which are computed by $H^{k+2}(M, \mathbb{Z})$. Thus, we see that extensions of the form above are classified topologically by $H^{k+2}(G, \mathbb{Z})$.

Now, in principle we are not yet done. We need to classify extensions with higher group structures, not just topological bundles. For 2-groups of the form

$$
1 \longrightarrow \mathrm{B} U(1) \longrightarrow \tilde{G}_{1} \longrightarrow G \longrightarrow 1,
$$

this second step was performed in [6] [theorem 99], [41][theorem 5.1.29]. An analogous analysis for more general cases is beyond the scope of this paper, see instead [41] [theorem 4.4.36]. See also [134] for a discussion of bundles of 2-groups, [39, 40] for a discussion of more general bundles of higher groups, and [7][section 3] for a discussion of the relationship between 2-groups and 1-gerbes.

Briefly, the result of the second step is that for $G$ a compact Lie group of dimension greater than zero, extensions of $G$ by any flavor of $\mathbf{B}^{k} U(1)$ are classified by $H^{k+2}(G, \mathbb{Z})$, just as topological class of bundles. However, for $G$ finite, there is additiona 28 information in the second step, and classes of extensions with higher group structures are classified by group cohomology $H^{k+2}(G, U(1))$ (with trivial action on the coefficients).

As a sanity check, note in the special case that $k=1$, for $G$ of dimension greater than zero, the extensions are classified by $H^{3}(G, \mathbb{Z})$, as consistent with WZW models and ChernSimons theories, for example. For $k=1$, if $G$ is finite, the extensions are classified by $H^{3}(G, U(1))$, consistent with three-dimensional Dijkgraaf-Witten theory.

${ }^{27}$ For $\tilde{G}_{1, k}$ and $\tilde{G}_{0, k}$, this follows from [41] [theorem 4.4.36]. For $\tilde{G}_{c, k}$, this follows from unpublished work of D. Pavlov [28. The reader should also note that the geometric realization of $\mathbf{B}^{k} U(1)_{\text {conn }}$ forgets the differential forms data, and so loses quite a bit of information.

${ }^{28}$ If $G$ is finite, then for $k \geq 0, H^{k+2}(G, \mathbb{Z})$ vanishes, so the topological bundles are all trivial. However, one can still have a nontrivial higher group extension, as this encodes additional structure on top of the topological bundle. 


\section{References}

[1] D. Gaiotto, A. Kapustin, N. Seiberg, B. Willett, "Generalized global symmetries," arXiv: 1412.5148.

[2] D. Freed, "Higher algebraic structures and quantization," Comm. Math. Phys. 159 (1994) 343-398, hep-th/9212115.

[3] J. Baez, J. Huerta, "An invitation to higher gauge theory," arXiv: 1003.4485.

[4] H. Sati, "Geometric and topological structures related to M-branes," arXiv: 1001.5020 .

[5] H. Sati, U. Schreiber, J. Stasheff, " $L_{\infty}$ algebras and applications to string- and ChernSimons $n$-transport," arXiv: 0801.3480.

[6] C. Schommer-Pries, "Central extensions of smooth 2-groups and a finite-dimensional string 2-group," Geom. Topol. 15 (2011) 609-676, arXiv: 0911.2483.

[7] T. Nikolaus, K. Waldorf, "Lifting problems and transgression for non-abelian gerbes," Adv. Math. 242 (2013) 50-79, arXiv: 1112.4702.

[8] J. Baez, A. Crans, D. Stevenson, U. Schreiber, "From loop groups to 2-groups," Homology, Homotopy Appl. 9 (2007) 101-135, math/0504123.

[9] T. Nikolaus, C. Sachse, C. Wockel, "A smooth model for the String group," Int. Math. Res. Not. IMRN 16 (2013) 3678-3721, arXiv: 1104.4288.

[10] D. Yetter, "TQFT's from homotopy 2-types," Journal of Knot Theory and its Ramifications 2 (1993) 113-123.

[11] J. Martins, T. Porter, "On Yetter's invariants and an extension of the DijkgraafWitten invariant to categorical groups," Theor. Appl. Categor. 18 (2007) 118-150, math/0608484.

[12] H. Grosse, K.-G. Schlesinger, "Duals for non-abelian lattice gauge theories by categorical methods," Int. J. Theor. Phys. 40 (2001) 459-475.

[13] H. Pfeiffer, "Higher gauge theory and a non-abelian generalization of 2-form electrodynamics," hep-th/0304074.

[14] J. Fröhlich, J. Fuchs, I. Runkel, C. Schweigert, "Defect lines, dualities, and generalised orbifolds," arXiv: 0909.5013.

[15] N. Carqueville, I. Runkel, "Orbifold completion of defect bicategories," arXiv: 1210.6363. 
[16] N. Carqueville, A. R. Camacho, I. Runkel, "Orbifold equivalent potentials," arXiv: 1311.3354.

[17] I. Brunner, N. Carqueville, D. Plencner, "Orbifolds and topological defects," arXiv: 1307.3141.

[18] I. Brunner, N. Carqueville, D. Plencner, "Discrete torsion defects," Comm. Math. Phys. 337 (2015) 429-453, arXiv: 1404.7497.

[19] N. Carqueville, A. Q. Velez, "Calabi-Yau completions and orbifold equivalences," arXiv: 1509.00880 .

[20] J. Wang, Z.-C. Gu, X.-G. Wen, "Field theory representations of gauge-gravity symmetry-protected topological invariants, group cohomology and beyond," arXiv: 1405.7689.

[21] G. Moore, "Finite in all directions," hep-th/9305139.

[22] K. Waldorf, "A construction of string 2-group models using a transgression-regression technique," pp. 99-115 in Analysis, geometry, and quantum field theory, Contemp. Math., vol. 584, Amer. Math. Soc., Providence, RI, 2012.

[23] K. Waldorf, "Transgression to loop spaces and its inverse, II: gerbes and fusion bundles with connectin," arXiv: 1004.0031.

[24] J. Baez, A. Lauda, "Higher-dimensional algebra V: 2-groups," math/0307200, Theor. Appl. Categor. 12 (2004) 423-491.

[25] J. Baez, A. Baratin, L. Freidel, D. Wise, "Infinite-dimensional representations of 2groups," arXiv: 0812.4969, Mem. Amer. Math. Soc. 219 (2012) no. 1032.

[26] H. Sinh, Gr-categories, Université Paris VII doctoral thesis, 1975.

[27] N. Ganter, private communication.

[28] U. Schreiber, private communication.

[29] J.-L. Brylinski, Loop spaces, characteristic classes, and geometric quantization, Birkhäuser, Boston, 1993.

[30] E. Sharpe, "Discrete torsion," Phys. Rev. D68 (2003) 126003, hep-th/0008154.

[31] U. Bunke, T. Nikolaus, M. Völkl, "Differential cohomology theories as sheaves of spectra," arXiv: 1311.3188.

[32] J. Milnor, J. Stasheff, Characteristic classes, Princeton University Press, 1974. 
[33] D. Fiorenza, U. Schreiber, J. Stasheff, "Cech cocycles for differential characteristic classes - an infinity-Lie theoretic construction," arXiv: 1011.4735.

[34] P. Bressler, "The first Pontryagin class," Compositio Math. 143 (2007) 1127-1163.

[35] P. Bouwknegt, talk at String-Math 2011.

[36] M. Garcia-Fernandez, "Torsion-free generalized connections and heterotic supergravity," Comm. Math. Phys. 332 (2014) 89-115, arXiv: 1304.4294.

[37] D. Baraglia, P. Hekmati, "Transitive Courant algebroids, string structures and Tduality," arXiv: 1308.5159.

[38] H. Sati, U. Schreiber, J. Stasheff, "Twisted differential String and Fivebrane structures," Comm. Math. Phys. 315 (2012) 169-213, arXiv: 0910.4001.

[39] T. Nikolaus, U. Schreiber, D. Stevenson, "Principal $\infty$-bundles - general theory," arXiv: 1207.0248.

[40] T. Nikolaus, U. Schreiber, D. Stevenson, "Principal $\infty$-bundles - presentations," arXiv: 1207.0249.

[41] U. Schreiber, "Differential cohomology in a cohesive $\infty$-topos," arXiv: 1310.7930.

[42] D. Fiorenza, C. Rogers, U. Schreiber, "A higher Chern-Weil derivation of AKSZ sigma models," Int. J. Geom. Methods Mod. Phys. 10 (2013) 1250078, arXiv: 1108.4378.

[43] B. Rosenstein, A. Kovner, "Masslessness of photon and Goldstone theorem," Int. J. Mod. Phys. A6 (1991) 3559-3570.

[44] A. Kovner, B. Rosenstein, "New look at $\mathrm{QED}_{4}$ : the photon as a Goldstone boson and the topological interpretation of electric charge," Phys. Rev. D49 (1994) 5571-5582.

[45] A. Kovner, "Confinement, magnetic $\mathbb{Z}_{N}$ symmetry and low energy effective theory of gluodynamics," pp. 1777-1825 in At the frontier of particle physics, volume 3, ed. M. Shifman, World Scientific, 2001, hep-ph/0009138.

[46] C. K. Altes, A. Kovner, "Magnetic $\mathbb{Z}_{N}$ symmetry in hot QCD and the spatial Wilson loop," Phys. Rev. D62 (2000) 096008, hep-ph/0004052.

[47] A. Kapustin, N. Saulina, "Topological boundary conditions in abelian Chern-Simons theory," arXiv: 1008.0654.

[48] U. Schreiber, talk at Workshop on higher categories and their applications, Fields Institute, Toronto, January 2007. 
[49] M. Berasaluce-González, G. Ramírez, A. Uranga, "Antisymmetric tensor $\mathbb{Z}_{p}$ gauge symmetries in field theory and string theory," JHEP 1401 (2014) 059, arXiv: 1310.5582.

[50] M. Ando, E. Sharpe, "Two-dimensional topological field theories as taffy," Adv. Theor. Math. Phys. 15 (2011) 179-244, arXiv: 1011.0100.

[51] T. Pantev, E. Sharpe, "GLSM's for gerbes (and other toric stacks)," Adv. Theor. Math. Phys. 10 (2006) 77-121, hep-th/0502053.

[52] T. Pantev, E. Sharpe, "Notes on gauging noneffective group actions," hep-th/0502027.

[53] T. Pantev, E. Sharpe, "String compactifications on Calabi-Yau stacks," Nucl. Phys. B733 (2006) 233-296, hep-th/0502044.

[54] O. Aharony, N. Seiberg, Y. Tachikawa, "Reading between the lines of four-dimensional gauge theories," arXiv: 1305.0318.

[55] D. Gaiotto, G. Moore, A. Neitzke, "Framed BPS states," arXiv: 1006.0146.

[56] S. Hellerman, E. Sharpe, "Sums over topological sectors and quantization of FayetIliopoulos parameters," Adv. Theor. Math. Phys. 15 (2011) 1141-1199, arXiv: 1012.5999.

[57] S. Hellerman, A. Henriques, T. Pantev, E. Sharpe, M. Ando, "Cluster decomposition, T-duality, and gerby CFT's," Adv. Theor. Math. Phys. 11 (2007) 751-818, hep-th/0606034.

[58] E. Sharpe, "Decomposition in diverse dimensions," Phys. Rev. D90 (2014) 025030, arXiv: 1404.3986.

[59] L. Anderson, B. Jia, R. Manion, B. Ovrut, E. Sharpe, "General aspects of heterotic string compactifications on stacks and gerbes," arXiv: 1307.2269.

[60] E. Andreini, Y. Jiang, H.-H. Tseng, "On Gromov-Witten theory of root gerbes," arXiv: 0812.4477 .

[61] E. Andreini, Y. Jiang, H.-H. Tseng, "Gromov-Witten theory of product stacks," arXiv: 0905.2258.

[62] E. Andreini, Y. Jiang, H.-H. Tseng, "Gromov Witten theory of root gerbes I: structure of genus 0 moduli spaces," arXiv: 0907.2087.

[63] E. Andreini, Y. Jiang, H.-H. Tseng, "Gromov-Witten theory of banded gerbes over schemes," arXiv: 1101.5996.

[64] A. Gholampour, H.-H. Tseng, "On Donaldson-Thomas invariants of threefold stacks and gerbes," Proc. Amer. Math. Soc. 141 (2013) 191-203. 
[65] H.-H. Tseng, "Notes on orbifold Gromov-Witten theory," proceedings of the Fifth International Congress of Chinese Mathematicians, AMS/IP Stud. Adv. Math. 51, part 1, volume 2, pp. 327-340.

[66] A. Caldararu, J. Distler, S. Hellerman, T. Pantev, E. Sharpe, "Non-birational twisted derived equivalences in abelian GLSMs," Comm. Math. Phys. 294 (2010) 605-645, arXiv: 0709.3855.

[67] K. Hori, "Duality in two-dimensional $(2,2)$ supersymmetric non-abelian gauge theories," JHEP 1310 (2013) 121, arXiv: 1104.2853.

[68] J. Halverson, V. Kumar, D. Morrison, "New methods for characterizing phases of 2d supersymmetric gauge theories," JHEP 1309 (2013) 143, arXiv: 1305.3278.

[69] E. Sharpe, "A few recent developments in $2 \mathrm{~d}(2,2)$ and $(0,2)$ theories," contribution to the proceedings of String-Math 2014, arXiv: 1501.01628.

[70] R. Dijkgraaf, E. Witten, "Topological gauge theories and group cohomology," Comm. Math. Phys. 129 (1990) 393-429.

[71] M. Douglas, "D-branes and discrete torsion," hep-th/9807235.

[72] M. Douglas, B. Fiol, "D-branes and discrete torsion 2," JHEP 0509 (2005) 053, hep-th/9903031.

[73] P. Aspinwall, "A note on the equivalence of Vafa's and Douglas's picture of discrete torsion," JHEP 0012 (2000) 029, hep-th/0009045.

[74] T. Pantev, private communication.

[75] E. Sharpe, "Analogues of discrete torsion for the M theory three-form," Phys. Rev. D68 (2003) 126004, hep-th/0008170.

[76] S. Monnier, "Higher abelian Dijkgraaf-Witten theory," arXiv: 1502.04706.

[77] E. Witten, "Nonabelian bosonization in two dimensions," Comm. Math. Phys. 92 (1984) 455-472.

[78] D. Fiorenza, H. Sati, U. Schreiber, "A higher stacky perspective on Chern-Simons theory," pp. 153-211 in Mathematical aspects of quantum field theories, ed. D. Calaque, T. Strobl, Springer, 2015, arXiv: 1301.2580.

[79] D. Gepner, E. Witten, "String theory on group manifolds," Nucl. Phys. B278 (1986) 493-549.

[80] U. Schreiber, "AQFT from n-functorial QFT," Comm. Math. Phys. 291 (2009) 357401, arXiv: 0806.1079. 
[81] N. Ganter, M. Kapranov, "Representation and character theory in 2-categories," Adv. Math. 217 (2008) 2268-2300, math/0602510.

[82] N. Ganter, "Categorical tori," arXiv: 1406.7046.

[83] N. Epa, private communication.

[84] G. Moore, N. Seiberg, "Taming the conformal zoo," Phys. Lett. B220 (1989) 422-430.

[85] J. Lurie, "On the classification of topological field theories," available at http://www.math.harvard.edu/ lurie/papers/cobordism.pdf

[86] D. Fiorenza, A. Valentino, "Boundary conditions for topological quantum field theories, anomalies and projective modular functors," arXiv: 1409.5723.

[87] D. Freed, "The cobordism hypothesis," arXiv: 1210.5100.

[88] D. Freed, C. Teleman, "Relative quantum field theory," Comm. Math. Phys. 326 (2014) 459-476, arXiv: 1212.1692.

[89] D. Freed, "Anomalies and invertible field theories," Proc. Symp. Pure Math. 88 (2014) 25-46, arXiv: 1404.7224.

[90] S. Weinberg, The quantum theory of fields, volume II: modern applications, Cambridge University Press, 1996.

[91] C. Itzykson, J.-B. Zuber, Quantum field theory, McGraw-Hill, 1980.

[92] L. Faddeev, "Operator anomaly for the Gauss law," Phys. Lett. 145B (1984) 81-84.

[93] L. Faddeev, S. Shatashvili, "Algebraic and Hamiltonian methods in the theory of nonabelian anomalies," Theor. Math. Phys. 60 (1985) 770-778.

[94] J. Mickelsson, "Chiral anomalies in even and odd dimensions," Comm. Math. Phys. 97 (1985) 361-370.

[95] A. C Davis, J. A. Gracey, A. J. Macfarlane, "Anomalous current algebras in the Skyrme model or chiral $G \times G$ with Wess-Zumino term," Phys. Lett. B194 (1987) 415-419.

[96] D. Freed, "Pions and generalized cohomology," hep-th/0607134.

[97] E. Witten, "Global aspects of current algebra," Nucl. Phys. B223 (1983) 422-432.

[98] L.-L. Chau, M.-L. Ge, Y.-S. Wu, "Kac-Moody algebra in the self-dual Yang-Mills equation," Phys. Rev. D25 (1982) 1086-1094.

[99] L.-L. Chau, Y.-S. Wu, "More about hidden symmetry algebra for the self-dual YangMills equation," Phys. Rev. D26 (1982) 3581-3592. 
[100] L.-L. Chau, M.-L. Ge, A. Sinha, Y.-S. Wu, "Hidden symmetry algebra for the self-dual Yang-Mills equation," Phys. Lett. B121 (1983) 391-396.

[101] L. Dolan, "A new symmetric group of real self-dual Yang-Mills theory," Phys. Lett. B113 (1982) 387-390.

[102] L. Crane, "Action of the loop group on the self dual Yang-Mills equation," Comm. Math. Phys. 110 (1987) 391-414.

[103] T. He, P. Mitra, A. Strominger, "2d Kac-Moody symmetry of 4d Yang-Mills theory," arXiv: 1503.02663.

[104] T. Adamo, E. Casali, "Perturbative gauge theory at null infinity," arXiv: 1504.02304.

[105] C. Vafa, E. Witten, "A strong coupling test of S duality," Nucl. Phys. B431 (1994) 3-77, hep-th/9408074.

[106] H. Nakajima, "Instantons on ALE spaces, quiver varieties, and Kac-Moody algebras," Duke Math. J. 76 (1994) 365-416.

[107] M. Henneaux, V. Lekeu, "Kac-Moody and Borcherds symmetries of six-dimensional chiral supergravity," arXiv: 1502.00518.

[108] B. Julia, "Group disintegrations," in Superspace and supergravity (Nuffield Gravity Workshop, Cambridge, England, June 22 - July 12, 1980), eds. S. W. Hawking and M. Rocek, Cambridge University Press, Cambridge, 1981.

[109] B. Julia, "Infinite Lie algebras in physics," in Current problems in particle physics, unified theories and beyond (John Hopkins University workshop, 1984).

[110] H. Nicolai, "A hyperbolic Lie algebra from supergravity," Phys. Lett. B276 (1992) 333-340.

[111] B. Julia, "Dualities in the classical supergravity limits: dualizations, dualities, and a detour via $(4 k+2)$-dimensions," pp. 121-139 in Strings, branes, and dualities (Cargese 1997), hep-th/9805083.

[112] A. Vilenkin, E. Shellard, Cosmic strings and other topological defects, Cambridge University Press, Cambridge, 1994.

[113] J. Preskill, L. Krauss, "Local discrete symmetry and quantum mechanical hair," Nucl. Phys. B341 (1990) 50-100.

[114] A. Schwarz, "Field theories with no local conservation of the electric charge," Nucl. Phys. B208 (1982) 141-158. 
[115] A. Schwarz, Y. Tyupkin, "Grand unification and mirror particles," Nucl. Phys. B209 (1982) 427-432.

[116] M. Bucher, H.-K. Lo, J. Preskill, "Topological approach to Alice electrodynamics," Nucl. Phys. B386 (1992) 3-26.

[117] W.-K. Tung, Group theory in physics, World Scientific, Philadelphia, PA, 1985.

[118] J.-L. Brylinski and D. McLaughlin, "The geometry of degree-four characteristic classes and of line bundles on loop spaces I," Duke Math. J. 75 (1994) 603-638.

[119] J. Distler, E. Sharpe, "Heterotic compactifications with principal bundles for general groups and general levels," Adv. Theor. Math. Phys. 14 (2010) 335-398, hep-th/0701244.

[120] S. J. Gates, Jr., W. Siegel, "Leftons, rightons, nonlinear sigma models, and superstrings," Phys. Lett. B206 (1988) 631-638.

[121] D. Depireux, S. J. Gates, Jr., Q-H. Park, "Lefton-righton formulation of massless Thirring models," Phys. Lett B224 (1989) 364-372.

[122] S. Bellucci, D. Depireux, S. J. Gates, Jr., "(1,0) Thirring models and the coupling of spin-zero fields to the heterotic string," Phys. Lett B232 (1989) 67-74.

[123] S. J. Gates, Jr., S. Ketov, S. Kuzenko, O. Soloviev, "Lagrangian chiral coset construction of heterotic string theories in (1,0) superspace," Nucl. Phys. B362 (1991) 199-231.

[124] S. J. Gates, Jr., "Strings, superstrings, and two-dimensional lagrangian field theory," pp 140-184 in Functional integration, geometry, and strings, proceedings of the XXV Winter School of Theoretical Physics, Karpacz, Poland (Feb. 1989), ed. Z. Haba, J. Sobczyk, Birkhauser, 1989.

[125] L. Alvarez-Gaumé, P. Ginsparg, "The structure of gauge and gravitational anomalies," Ann. Phys. 161 (1985) 423-490.

[126] N. Ganter, "Hecke operators in equivariant elliptic cohomology and generalized Moonshine," pp. 173-209 in Groups and symmetries, CRM Proc. Lecture Notes vol. 47, Amer. Math. Soc., Providence, Rhode Island, 2009.

[127] T. Eguchi, H. Ooguri, Y. Tachikawa, "Notes on the K3 surface and the Mathieu group $M_{24}$," Exper. Math. 20 (2011) 91-96, arXiv: 1004.0956.

[128] T. Eguchi, K. Hikami, "Enriques moonshine," J. Phys. A46 (2013) 312001, arXiv: 1301.5043. 
[129] T. Eguchi, K. Hikami, "N=2 moonshine," Phys. Lett. B717 (2012) 266-273, arXiv: 1209.0610.

[130] A. Taormina, K. Wendland, "The overarching finite symmetry group of Kummer surfaces in the Mathieu group $M_{24}$," JHEP 1308 (2013) 125, arXiv: 1107.3834.

[131] A. Taormina, K. Wendland, "The symmetries of the tetrahedral Kummer surface in the Mathieu group $M_{24}, "$ arXiv: 1008.0954.

[132] J. Conway, N. Elkies, J. Martin, "The Mathieu group $M_{12}$ and its pseudogroup extension $M_{13}$," Experiment. Math. 15 (2006) 223-236.

[133] G. Moore, N. Seiberg, "Classical and quantum conformal field theory," Comm. Math. Phys. 123 (1989) 177-254.

[134] T. Bartels, "Higher gauge theory I: 2-bundles," math/0410328. 